\title{
Application of Quality by Design Approach to Develop Novel Optimized Self-emulsifying Drug Delivery System of Ezetimibe for Treatment of Poorly Water-soluble Antilipidemic Drug to Enhance its Bioavailability by Using D-optimal Mixture Design
}

\author{
Mukesh S Patel ${ }^{1 *}$, Alpesh D Patel ${ }^{1}$ and Mayur Patel ${ }^{2}$ \\ ${ }^{1}$ Department of Pharmaceutics, Shri B.M. Shah College of Pharmaceutical \\ Education and Research, Gujarat, India \\ ${ }^{2}$ Research Scholar, Gujarat Technological University, Ahmedabad, Gujarat, India \\ *Corresponding Author: Mukesh S Patel, Department of Pharmaceutics, Shri B.M. \\ Shah College of Pharmaceutical Education and Research, Gujarat, India.
}

Received: June 28, 2021

Published: July 14, 2021

(C) All rights are reserved by Mukesh S Patel., et al.

\begin{abstract}
The purpose of the research aimed to develop and optimize a novel dosage form of the self-emulsifying drug delivery systems of the Ezetimibe using quality by design (QbD) to enhancing its solubility. We performed solubility and emulsification tests to select a suitable initial risk assessment facilitated the selection of Capmul PG-8 (oil), Kolliphor RH40 (surfactant), Transcutol P (co-surfactant) as Critical Material Attributes (CQA) for the formulation of SEDDS. A d-optimal mixture design use to optimize the concentration of components used in the SEDDS formulation to achieve excellent physicochemical characteristics. Sixteen distinct formulations were prepared and assessed to check the model fit. It earmarked the patient-centric quality target product profile and CQAs. QbD resulted in a powerful and economical technique for improving the bioavailability of the medication, as confirmed by the portrayal investigations of the optimized batch. It saw a great arrangement among anticipated and exploratory qualities for mean droplet size, drug content, emulsification time, and percentage of medication delivered in 20 minutes. Optimized SEDDS formulation showed a smaller droplet size of $56.25 \mathrm{~nm}$, PDI 0.230, and zeta potential -13.6 mV. To sum up, we successfully used QbD oriented development of novel Ezetimibe-loaded SEDDS formulation using the d-optimal mixture design to improve the oral absorption of poorly watersoluble drugs.
\end{abstract}

Keywords: Ezetimibe; SEDDS; QbD; d-Optimal Mixture Design; Risk Estimation Matrix

\section{Abbreviations}

SEDDS: Self-emulsifying Drug Delivery Systems; QbD: Quality by Design (QbD); CQAs: Critical Quality Attributes; CPPs: Critical Process Parameters; CMAs: Critical Material Attributes; HLB: Hydrophilic Lipophilic Balance; ANOVA: Analysis of Variance; QTPP: Quality Target Product Profile

\section{Introduction}

The oral route is the most accessible and most convenient route for non-invasive administration. However, the solubility of the orally administered drug is significant challenges of the pharma- ceutical industry as nearly $35-40 \%$ of newly launched drugs possess low aqueous solubility, which leads to their poor dissolution and low bioavailability resulting in high intra and inter-subject variability and lack of dose proportionality [1]. Solubility is the significant interaction for the vast majority of the drugs to solubilize in an offered solvent to give a homogenous arrangement. The more noteworthy the solvency of the drug, the more prominent will be the total dissolution showing a wanted pharmacological response [2].

Many new methods enhance the solubility of inadequately soluble drugs like Solid dispersion, Complexation, Particle size reduc-

Citation: Mukesh S Patel., et al. "Application of Quality by Design Approach to Develop Novel Optimized Self-emulsifying Drug Delivery System of Ezetimibe for Treatment of Poorly Water-soluble Antilipidemic Drug to Enhance its Bioavailability by Using D-optimal Mixture Design". Acta Scientific Pharmaceutical Sciences 5.8 (2021): 20-42. 
tion, co-solvency, etc. Among these, Self-Emulsifying Drug Delivery System (SEDDS) is gaining popularity for improving the solubility of lipophilic drugs [3]. SEDDS characterized as isotropic combinations of at least one hydrophilic solvent and cosolvent/surfactants that can form fine oil-in-water $(\mathrm{o} / \mathrm{w})$ microemulsions upon mild fomentation by dilution in aqueous media, for example, G.I. fluids. Later on, the formed emulsion absorbs through the lymphatic pathway. Therefore, it would improve the oral bioavailability of drugs in SEDDS to bypass the liver's first-pass effect. Thus, SEDDS has become a vital strategy to increase the oral bioavailability of poorly water-soluble drugs. Moreover, there is no fluid phase in SEDDS; it is a homogeneous framework, thus being appropriate for huge scope creation and steadier than traditional emulsion $[4,5]$.

SEDDS has proven to be promising in facilitating the oral bioavailability of poorly water-soluble drugs. Self-emulsifying formulations spread promptly in the G.I. path, the motility of the stomach and the intestine provide necessary agitation for self-emulsification. SEDDS commonly produce emulsions with the droplet size somewhere in the range of 100 to $300 \mathrm{~nm}[6,7]$. In comparison, SEDDS form clear microemulsions with a droplet size of $50 \mathrm{~nm}$. Compared with emulsions, which are delicate and metastable scattered forms, SEDDS are physically steady formulations that are not difficult to make. Consequently, for a lipophilic drug that displays dissolution rate-restricted absorption, these systems may offer an improvement in the rate and degree of absorption and create a more reproducible blood time profile [8].

The pharmaceutical business is highly controlled, and the authoritative regulatory bodies control it. The regulatory agencies emphasize the quality of pharmaceutical products. However, it can design quality, and most of the quality deficiency occurs when the process was designed and developed. The quality expert Joseph Moses Juran introduced Quality by Design and its application in product development. The principles of QbD have been used in every industry to improve the quality of the product and process. In addition, all-around controlled and reproducible outcomes secure to accomplish the necessary therapeutic objectives of the formulation by the exact strategy called quality by design [3,8-10].

In the present study, Ezetimibe-loaded SEDDS formulations were designed and optimized by a Design of Experiments approach called D-optimal mixture design. It is a mathematical technique used to pick the framework's parts (exploratory inputs), which will apply the most unimportant impact on the product inconstancy. The Mixture Design created using the JMP $®$ trial Software 16 ver- sion (SAS Institute, Cary, NC, USA) [11]. The Critical Material Attributes (CMAs) decided for the examination were oil (Capmul PG-8), surfactant (Kolliphor RH 40), and co-surfactant (Transcutol P), and the Critical Process Parameters (CPPs) were conditions like blending speed, sort of stirrer, and the mixing temperature. In addition, CQAs chose to enhance globule size, emulsification time, drug content, and in vitro drug discharge study $[9,12]$.

\section{Materials and Methods}

Ezetimibe was acquired as a blessing sample from Lupin Ltd, Ahmedabad. Labrasol, Transcutol CG, Transcutol P, MCT, Labrafac P, Capryol 90, Labrafac PG, Capmul PG 8 N.F., and Labrafil M 2125 were acquired Gattefosse, France. Capex 200 P, MCT, Capmul MCM, and Captex 355 was received from Abitech corporation, U.S. Acryl EL 135, Acrysol K 160, Acrysol K 140, and K150 were purchased from Corel chemical Pvt Ltd Ahmedabad, India. Sunflower oil, Seasame oil, Canola oil, and Olive oil were procured from Kiran oil Ltd, Ahmedabad. Flaxseed oil/ Linseed oil, Soyabean oil, and Coconut oil were acquired from Kush protein Ltd, Anand. Sefsol 218 (Monocaprylic Ester) was obtained from Nikko Chemical Co. Ltd, Japan. Kolliphor RH40 was supplied from BASF, Germany. Tween 80, Span 80, Tween 20, and Polyethylene glycol was purchased from finer chemical limited, Ahmedabad, India, and All the materials and reagents utilized for the research project were of the analytical grade. Deionized water was used throughout the study.

Defining quality target product profile (QTPP) and critical quality attributes (CQAs)

QTPP approach establishes a planning system for item improvement, and it begins with "plan as a primary concern" to ensure product efficacy and safety. The QTPP is a product sketch that precise the characteristics expected during the development of the product to respond to the therapeutic objective of the medication. At last, the quality objective item profile frames the root for the drug [13]. Ultimately quality target product profile forms the core for the development of the product. Through the QbD approach, the product development advocates defining the QTPP, and it is one of the prerequisites to deliver therapeutic benefits as per the label claim. The QTPP for liquid SEDDS was determined based on the patient-centric (emulsification time, globule size, drug content, drug release) and product-centric (PDI, zeta potential) drug product quality attributes. The CQAs identified from QTPP were interlinked to give the desired quality, safety, and efficacy to the product, showing noticeable changes when QTPP is altered $[6,9,13]$. The QTPP and CQAs were listed in table 1 and 2, correspondingly.

Citation: Mukesh S Patel., et al. "Application of Quality by Design Approach to Develop Novel Optimized Self-emulsifying Drug Delivery System of Ezetimibe for Treatment of Poorly Water-soluble Antilipidemic Drug to Enhance its Bioavailability by Using D-optimal Mixture Design". Acta Scientific Pharmaceutical Sciences 5.8 (2021): 20-42. 
Application of Quality by Design Approach to Develop Novel Optimized Self-emulsifying Drug Delivery System of Ezetimibe for Treatment of Poorly Water-soluble Antilipidemic Drug to Enhance its Bioavailability by Using D-optimal Mixture Design

\begin{tabular}{|c|c|c|}
\hline QTPP elements & Target & Justification \\
\hline Dosage type & Lipid-based formulation & Enhancement of Bioavailability of poorly water-soluble drugs \\
\hline Dosage form & Capsule & $\begin{array}{l}\text { Ease of administration and Formulated as hard gelatin capsules for } \\
\text { improved patient consistency, compactness, and assembling ease }\end{array}$ \\
\hline Dosage strength & $100 \mathrm{mg}$ & Required to show the therapeutic action at the target site \\
\hline Route of administration & Oral & Most convenient route for patients \\
\hline Product Quality Attributes & $\begin{array}{c}\text { Physical attributes } \\
\text { Droplet size } \\
\text { Transmittance } \\
\text { PDI and Zeta potential } \\
\text { Emulsification efficiency } \\
\text { Drug content } \\
\text { Drug release }\end{array}$ & The formulation must meet the compendial quality standards \\
\hline Stability & $\begin{array}{l}\text { According to the states of ICH } \\
\text { Q1B Long term stability study. }\end{array}$ & $\begin{array}{l}\text { To assess the depredatory pattern of the Drug and Excipients used } \\
\text { in the formulation. }\end{array}$ \\
\hline
\end{tabular}

Table 1: Quality target product profile of Ezetimibe -SEDDS.

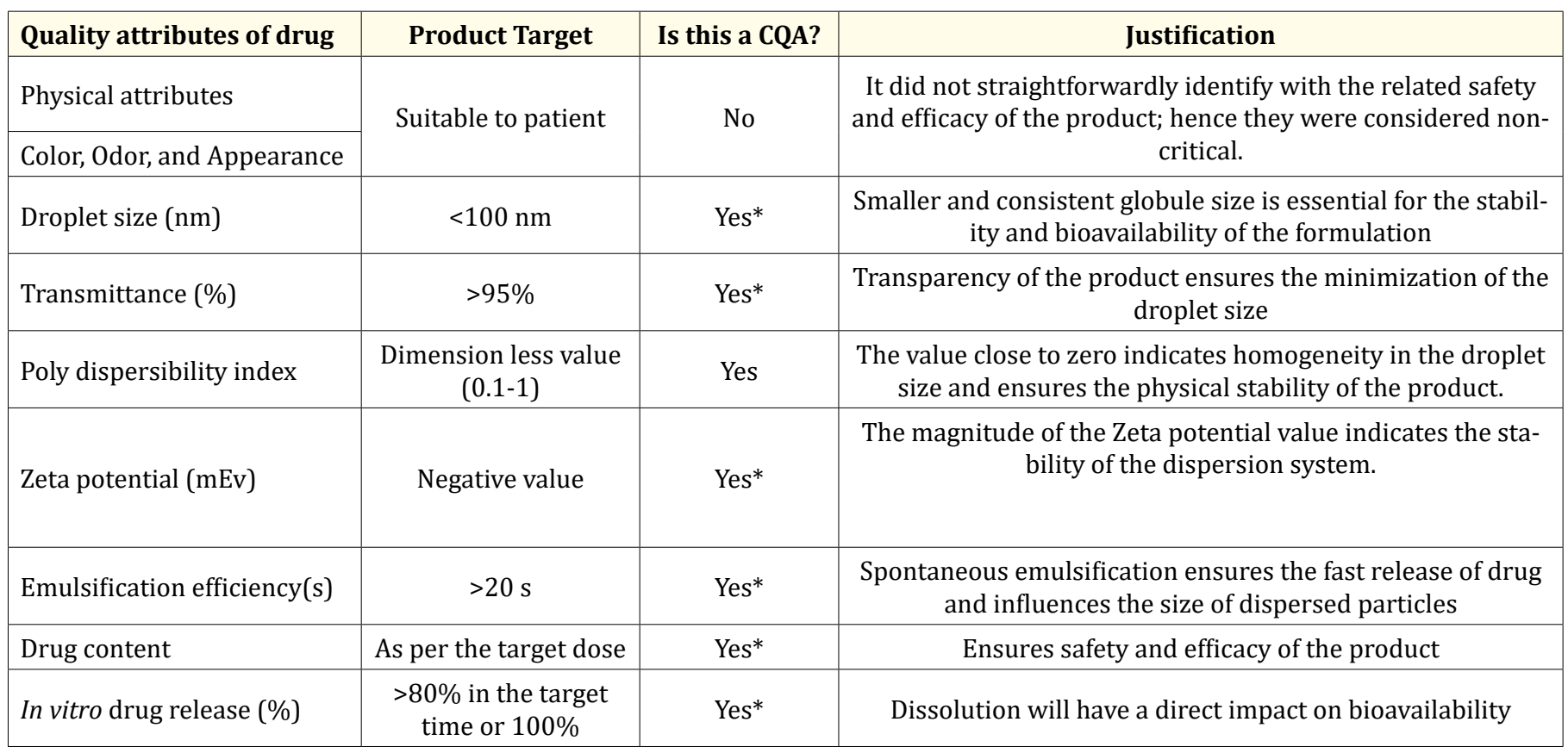

Table 2: Critical quality attributes (CQA) of SEDDS system with justification.

SEMDDS: Self-emulsifying drug delivery systems **CQAs considered as critical.

Citation: Mukesh S Patel., et al. "Application of Quality by Design Approach to Develop Novel Optimized Self-emulsifying Drug Delivery System of Ezetimibe for Treatment of Poorly Water-soluble Antilipidemic Drug to Enhance its Bioavailability by Using D-optimal Mixture Design". Acta Scientific Pharmaceutical Sciences 5.8 (2021): 20-42. 
Application of Quality by Design Approach to Develop Novel Optimized Self-emulsifying Drug Delivery System of Ezetimibe for Treatment of Poorly Water-soluble Antilipidemic Drug to Enhance its Bioavailability by Using D-optimal Mixture Design

\section{Risk assessment}

The dosage form development under the QbD framework involves evaluating material and process attributes that have a more vital influence on the quality of the drug. Risk assessment is a combined effort to identify and assess the factors that may impact the product's CQAs. The risk assessment tools help identify and mitigate the risks and prioritize the risk as high, medium, or low based on the impact level. Table 3 represents the risk estimation matrix for Ezetimibe SEDDS. The two qualitative tools used in the present study were the Fishbone diagram and the Risk Estimation Matrix table. The Fishbone diagram (Figure 1) constructed using JMP ${ }^{\circledR}$ Software 16 version (Academic license from SAS, SAS Institute, Cary, NC, USA). The Fishbone diagram depicts the cause and subcauses affecting the CQAs, and the risk assessment matrix helps categorize the risk $[8,9,13,14]$.

\begin{tabular}{|l|c|c|c|c|c|c|}
\hline CMA /CPP CQAs & $\begin{array}{c}\text { API } \\
\text { Particle size }\end{array}$ & Oil & Surfactant & Cosurfactant & $\begin{array}{c}\text { Stirring } \\
\text { speed }\end{array}$ & Stirring time \\
temperature
\end{tabular}

Table 3: Risk Estimated Matrix.

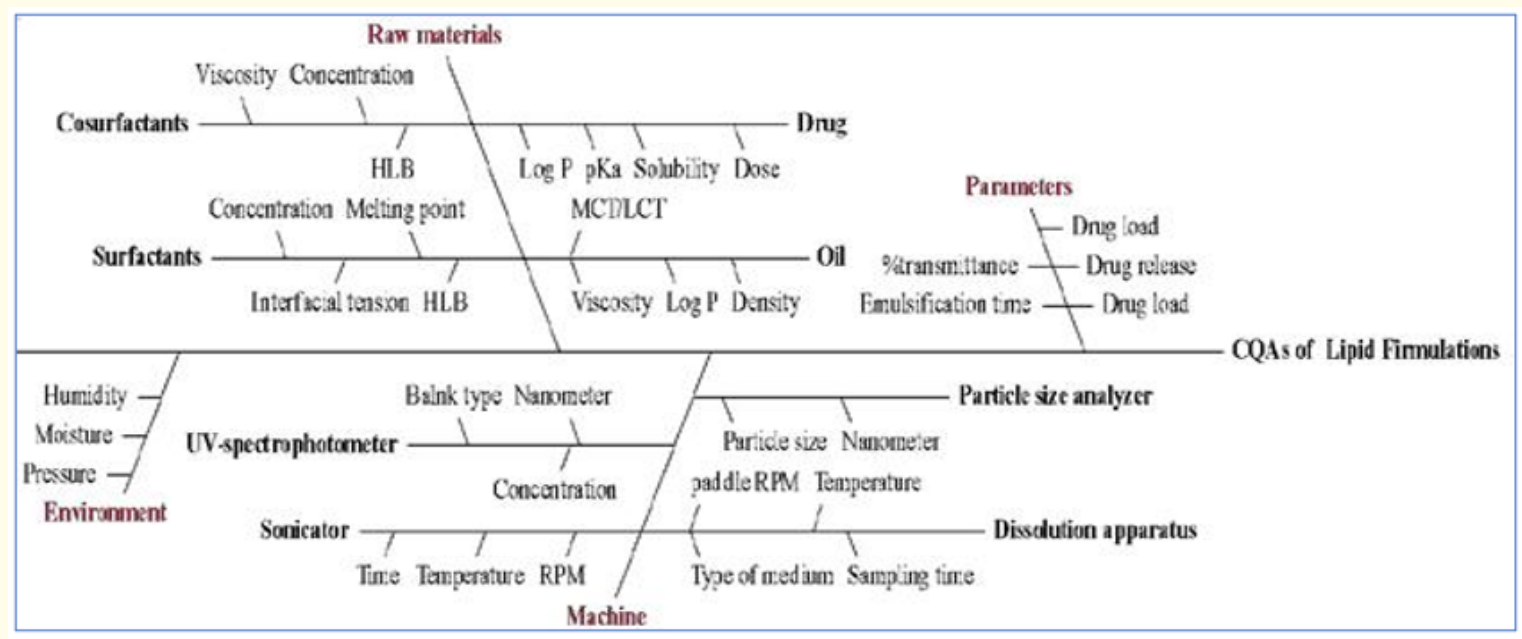

Figure 1: Fishbone diagram depicting causes and sub causes affecting Ezetimibe -SEDDS quality attributes. 
Application of Quality by Design Approach to Develop Novel Optimized Self-emulsifying Drug Delivery System of Ezetimibe for Treatment of Poorly Water-soluble Antilipidemic Drug to Enhance its Bioavailability by Using D-optimal Mixture Design

24

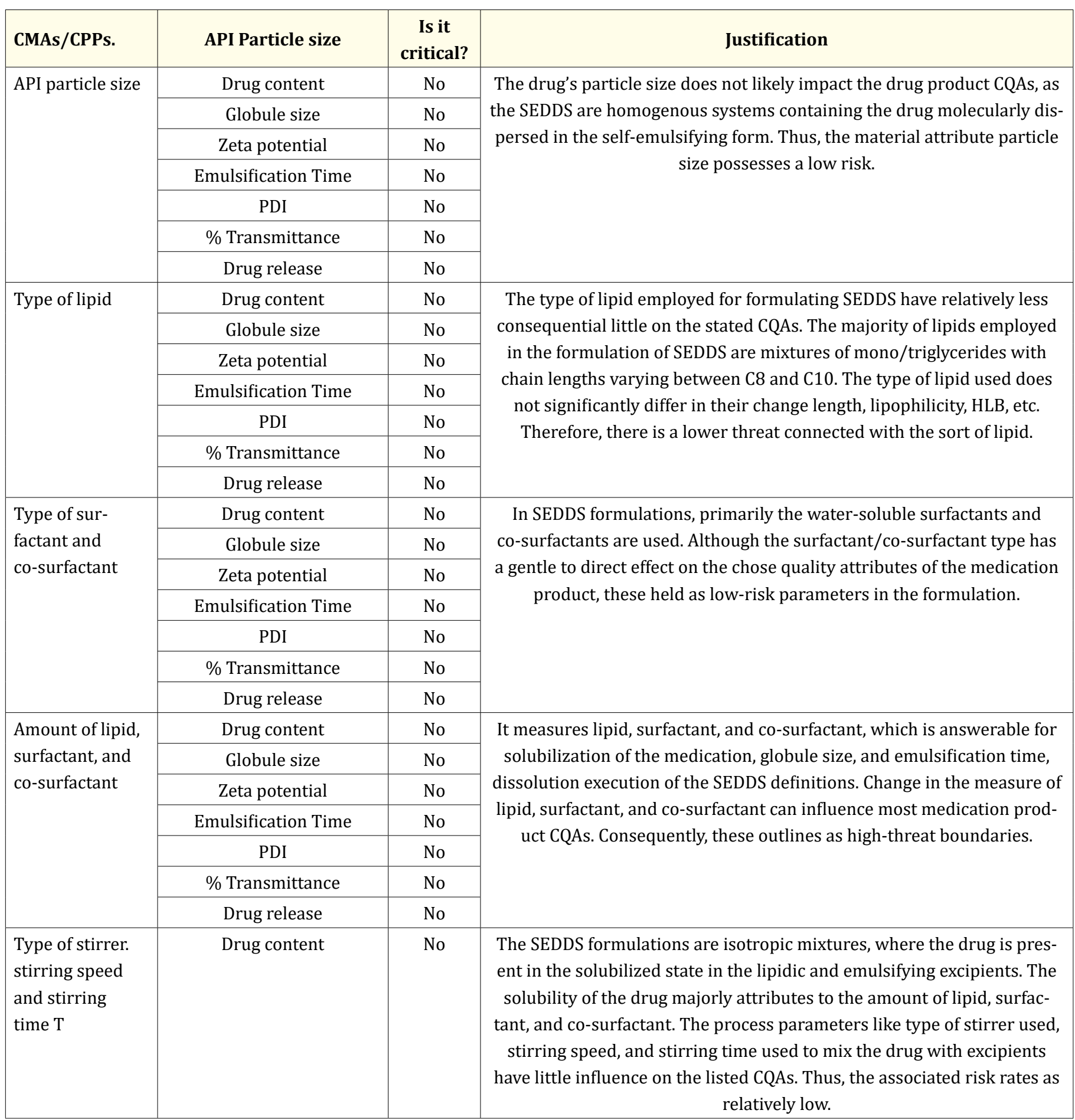

Table 4: Justification of risk allotment and identification of Critical Material Attributes and Critical Process Parameters. 
Application of Quality by Design Approach to Develop Novel Optimized Self-emulsifying Drug Delivery System of Ezetimibe for Treatment of Poorly Water-soluble Antilipidemic Drug to Enhance its Bioavailability by Using D-optimal Mixture Design

\begin{tabular}{|c|c|c|c|}
\hline \multicolumn{3}{|c|}{ Composition and limits of experimental domain } \\
\hline \multirow{2}{*}{ Critical Material Attributes (Factor) } & \multirow{2}{*}{ Role } & Low & Halues \\
\cline { 2 - 4 } & & 0.15 & 0.2 \\
\hline Capmul PG-8 & Mixture & 0.3 & 0.5 \\
\hline Kolliphor RH 40 & Mixture & 0.3 & 0.5 \\
\hline Transcutol P & Mixture & Lower Limit & Upper Limit \\
\hline Responses & Responses in mixture design. & 100 \\
\hline Drug release in 20 minutes (\%) & Goal & 80 & 100 \\
\hline \% Drug Content & Maximize & 95 & 60 \\
\hline Emulsification time (seconds) & Maximize & 20 & 100 \\
\hline Droplet size (nm) & Minimize & 50 & \\
\hline
\end{tabular}

Table 5: Mixture design components and response.

\begin{tabular}{|l|c|c|c|c|c|c|c|}
\hline $\begin{array}{l}\text { Formulation } \\
\text { Code }\end{array}$ & $\begin{array}{c}\text { Capmul } \\
\text { PG-8 }\end{array}$ & $\begin{array}{c}\text { Kolliphor } \\
\text { RH 40 }\end{array}$ & Transcutol P & $\begin{array}{c}\text { Formulation } \\
\text { Code }\end{array}$ & Capmul PG-8 & $\begin{array}{c}\text { Kolliphor } \\
\text { RH 40 }\end{array}$ & \begin{tabular}{c} 
Transcutol P \\
\hline F1
\end{tabular} \\
\hline F2 & 0.5 & 0.3 & F9 & 0.185 & 0.5 & 0.315 \\
\hline F3 & 0.15 & 0.35 & 0.5 & F10 & 0.2 & 0.44 & 0.36 \\
\hline F4 & 0.15 & 0.5 & 0.35 & F11 & 0.15 & 0.425 & 0.425 \\
\hline F5 & 0.165 & 0.404 & 0.431 & F12 & 0.166623 & 0.333377 & 0.5 \\
\hline F6 & 0.165 & 0.5 & 0.335 & F13 & 0.2 & 0.36 & 0.44 \\
\hline F7 & 0.185618 & 0.314382 & 0.5 & F14 & 0.185 & 0.462 & 0.353 \\
\hline F8 & 0.2 & 0.3 & 0.5 & F15 & 0.19 & 0.367 & 0.443 \\
\hline
\end{tabular}

Table 6: Composition of SEDDS as per the d-Optimal Mixture Design.

Preformulation study

Selection of oil

We selected oils based on the solubility of Ezetimibe, \% transparency, and ease of emulsification in various modified oils, surfactants, and Co-surfactants. Take $2 \mathrm{ml}$ of each element in screw cap vials with the known amount (100 mg) of the drug. A vortex mixture was utilized for the solubilization of the drug. Sealed vials were put on an isothermal mechanical shaker at $40 \pm 2^{\circ} \mathrm{C}$ for 48 hours. After balance, each test tube was centrifuged at 3000 rpm for 20 min using a centrifuge. The supernatant was separated through a membrane filter by utilizing a $0.45-\mu \mathrm{m}$ filter paper. The separate solution was appropriately diluted with methanol and examined in U.V. Spectrometers at $315 \mathrm{~nm}$. The amount of solubilized drug was determined using a standard equation. The results of solubility study data are depicted in figure $3 \mathrm{~A}$.

\section{Selection of surfactant}

We selected surfactants based on the solubility of the drug, \% transparency, and ease of emulsification. Solubility of the drug in surfactant was evaluated in a comparative way to portray in the above segment of oil $[7,15]$. It can screen the emulsification capacity of various surfactants in the oil phase. Briefly, take $0.5 \mathrm{ml}$ of surfactant and $0.5 \mathrm{ml}$ of oil mixed and heated at $50{ }^{\circ} \mathrm{C}$ for homogenization of components. Each $0.1 \mathrm{ml}$ mixture was diluted with the $50 \mathrm{ml}$ distilled water in a glass stopper conical flask. The ease of emulsification was determined by the number of flask inversion required for the homogeneous emulsion. A resulting emulsion was examined visually for the relative turbidity. An emulsion was allowed to stand for $2 \mathrm{hrs}$. And their transmittance was observed at $650 \mathrm{~nm}$ through a UV-1800 double beam spectrophotometer utilizing double distilled water as blank. Desired HLB value to form 
o/w SEDDS must be in the range of 12-18, and the surfactant was mainly selected based on this. Naked eyes further examined emulsification for any turbidity and phase separation. The emulsification ability of different surfactants exhibits figure 3B.

\section{Selection of cosurfactant}

We made a selection of cosurfactant based on the solubility of the drug, \% transparency, and ease of emulsification. First, the solubility of the drug in cosurfactant was examined as per mentioned procedure in the above selection of oil. Then, a mixture of co-surfactant, surfactant, and selected oil was prepared and analyzed as described in the above section of surfactants $[15,16]$. Emulsification ability appears in figure $3 \mathrm{C}$.

Drug excipient compatibility study using FTIR 8400 S spectrometer

The FTIR instruments dictated the recognition of pure drugs.
Ezetimibe mixed thoroughly with potassium bromide, an infrared transparent matrix, 1:10 (Drug: $\mathrm{KBr}$ ) proportion. The $\mathrm{KBr}$ discs were set up by compressing the Ezetimibe with $\mathrm{KBr}$. Spectra were obtained at a resolution from $4000 \mathrm{~cm}^{-1}-400 \mathrm{~cm}^{-1}$.

A FTIR-8400S spectrometer outfitted with attenuated total reflectance adornment use to get the infrared spectra of the drug in the isotropic combination of excipients, Analysis of pure drug, physical mixture of the medication with the other excipients. The formulation is lyophilized utilizing a freeze dryer, and FTIR studies have carried out diffuse reflectance spectroscopy FTIR with $\mathrm{KBr}$ disc. Every one of the samples dried using a vacuum before getting any spectra to eliminate the influence of residual moisture. For each range spectrum, eight scans were obtained at a resolution of 4 $\mathrm{cm}^{-1}$ from a frequency range of $4000-400 \mathrm{~cm}^{-1}$ and demonstrated in figure 2 .

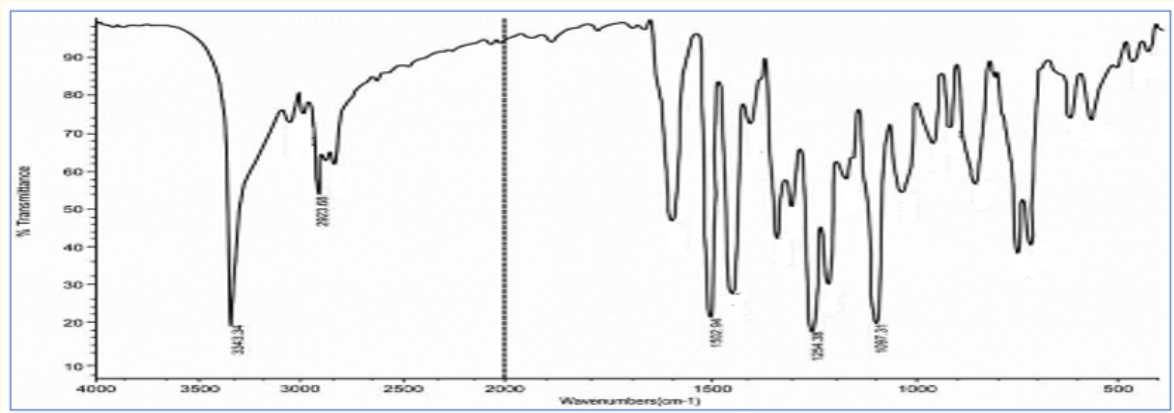

(A)

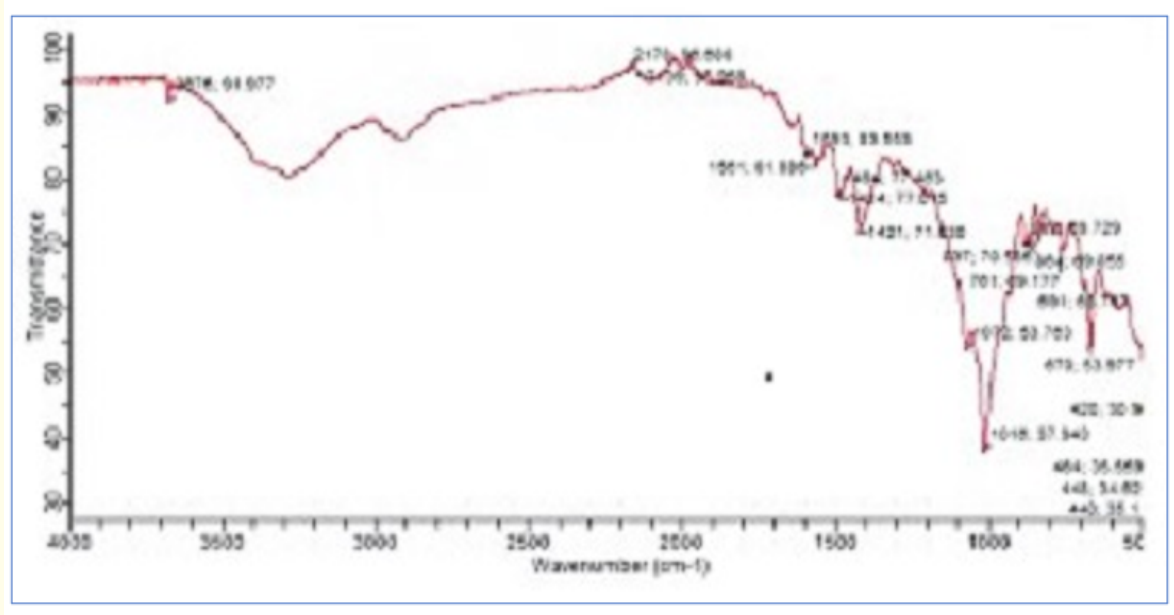

(B)

Figure 2: A). FTIR spectra of Ezetimibe. B). FTIR spectra of Ezetimibe loaded SEDDS formulation. 


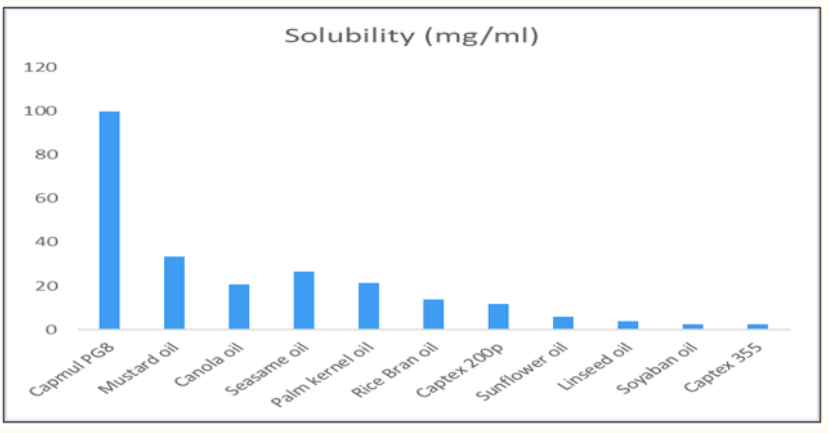

Figure 3A: Schematic diagram of Ezetimibe in different oils.

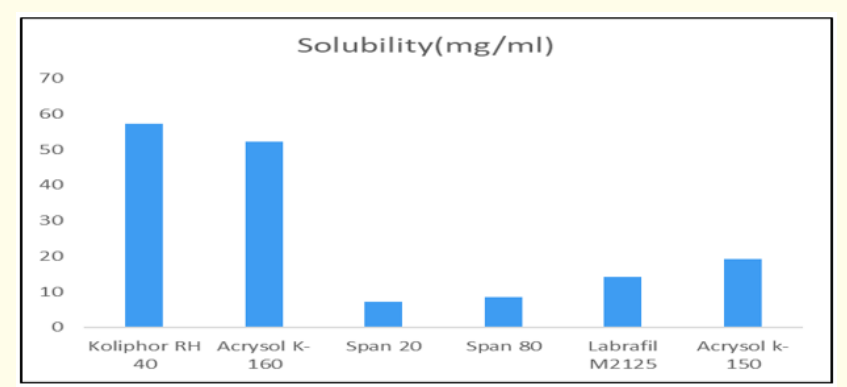

Figure 3B: Schematic diagram of Ezetimibe solubility in different surfactant.

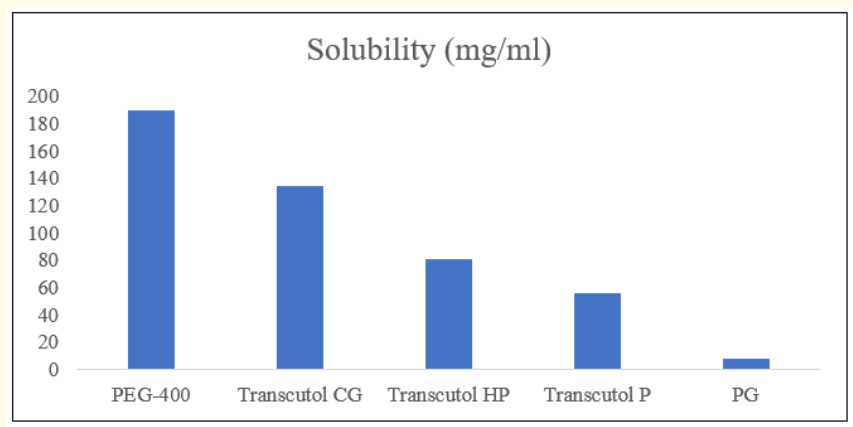

Figure 3C: Schematic diagram of Ezetimibe solubility in various co-surfactants.

Figure 3: Schematic diagram of Ezetimibe solubility in different oils, surfactants, and Co-surfactants.

\section{Preparation of SEDDS (Without Ezetimibe)}

The formulation consists of different ratios of oil (Capmul PG-8), surfactant (Kolliphor RH40), and cosurfactant (Transcutol P). First, the combination was combined by vortexing until a transparent solution was acquired. Then, the formulations were equilibrated at the surrounding temperature for $24 \mathrm{hrs}$. Finally, the formulations were examined for signs of turbidity or phase separation before self-emulsification.

\section{Construction of phase diagrams}

We constructed pseudo-ternary phase diagrams to obtain the appropriate components and their concentration ranges, resulting in a large existence area of the microemulsion. The microemulsion region was initially delineated by its isotropic nature and low viscosity to optimize the oil, surfactant, and co-surfactant phase concentration; different batches of varying concentrations were prepared and titrated with distilled water to appear turbidity. A two-dimensional ternary phase diagram can be set up by keeping the composition of one component fixed and varying the other two or using a constant ratio of surfactant to co-surfactant. Here, the ternary phase diagram was set up by utilizing a consistent surfactant ratio to co-surfactant $[7,8,15,16]$.

\section{Pseudo-ternary phase diagram for SEDDS}

Pseudo ternary phase diagrams have been built utilizing the chosen component, demonstrating the ingredients' amount. That can bring results in an enormous area of the microemulsion. These plots were constructed with the use of oil (Capmul PG-8), surfactant (Kolliphor RH40), co-surfactant (Transcutol P), and water with the help of water titration technique at room temperature. The surfactant and co-surfactants were combined in various proportions like $1: 1,1: 2$, and 2:1 accordingly. The oil and Smix were mixed, which varied from 9:1 to $1: 9$ parts. We created a ternary phase diagram with the help of an aqueous titration strategy. Every mixture was titrated with distilled water and stirred at room temperature to acquire equilibrium. Then, the mixture was visually evaluated for transparency. The ternary diagrams prepare using oil, surfactant, and co-surfactant; it considered the point showing the transparent and isotropic mixtures to be withinside the microemulsion area $[17,18]$. Pseudo ternary plots were constructed using Ternary Plot. com. Results of the phase diagram are stated in figure 4A-4C. 
$4(A)$

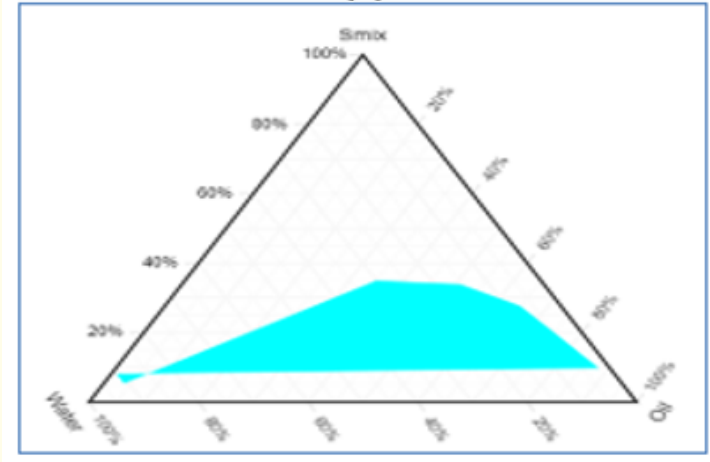

$4(C)$

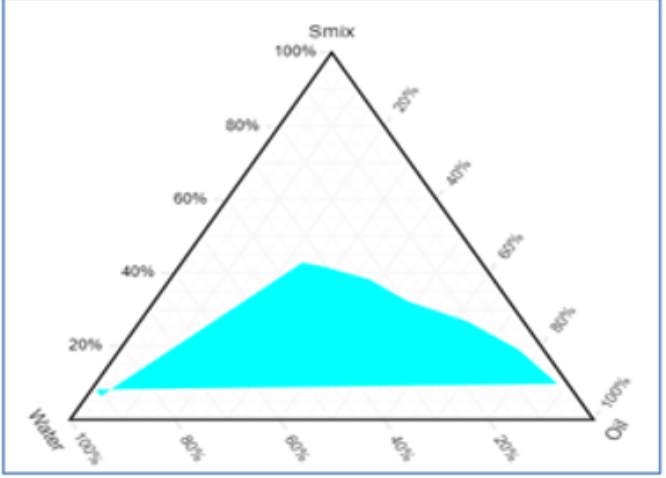

$4(\mathrm{E})$

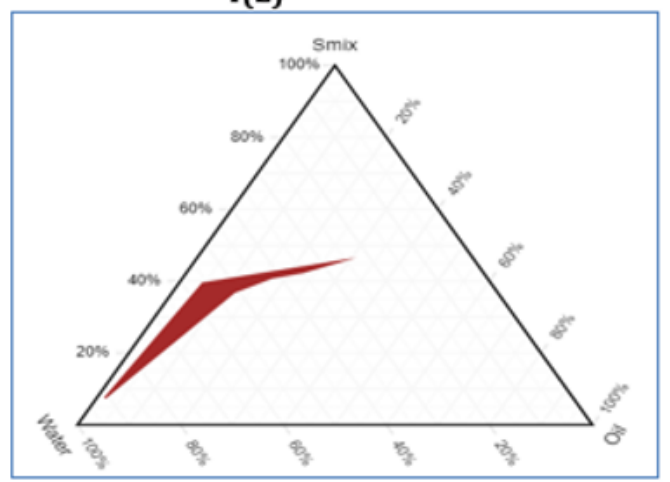

4(B)

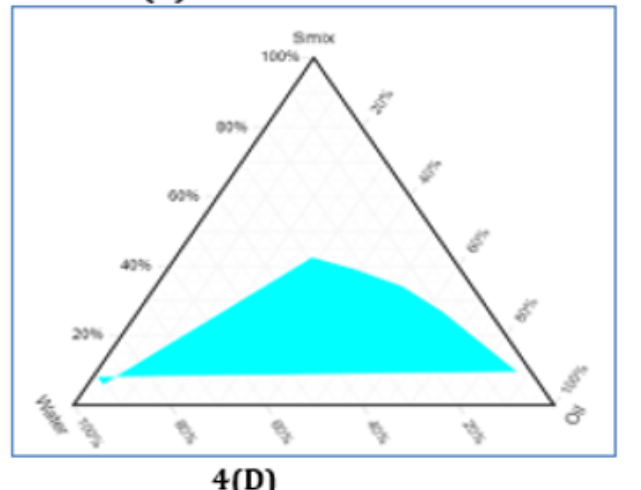

4(D)

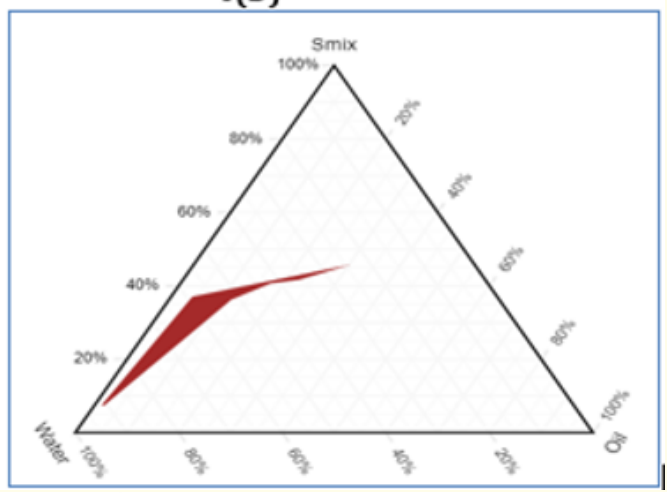

4(F)

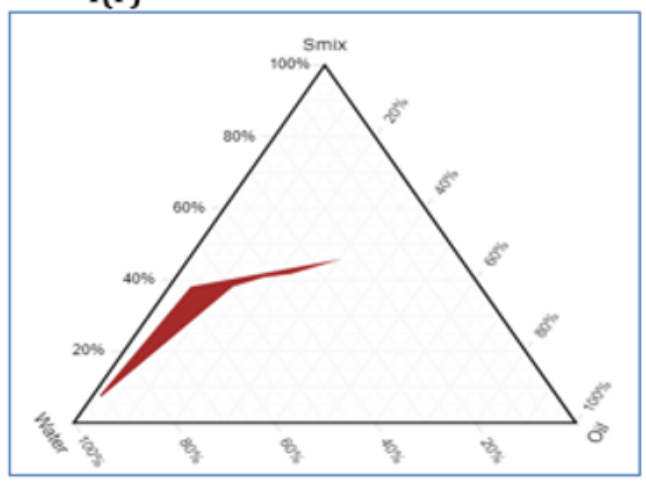

Figure 4: Pseudo-Ternary Phase diagram. A) Surfactant (Kolliphor RH40)/co-surfactant (Transcutol p) ratio (1:1) and oil (Capmul PG-8) B). Surfactant (Kolliphor RH40)/Co-surfactant (Transcutol P) ratio (2:1) and Oil (Capmul PG-8)) C). Surfactant (Kolliphor RH40)/Cosurfactant (Transcutol P) ratio (1:2) and Oil (Capmul PG-8) D). Surfactant (Kolliphor RH40)/co-surfactant (Transcutol p) ratio (1:1) and oil (Capmul PG-8) E). Surfactant (Kolliphor RH40)/co-surfactant (Transcutol p) ratio (2:1) and oil (Capmul PG-8) F). Surfactant (Kolliphor RH40)/Co-surfactant (Transcutol P) ratio (1:2) and Oil (Capmul PG-8).

\section{Formulation of SEDDS (With Ezetimibe)}

The formulations were frame-up by dissolving $25 \mathrm{mg}$ of Ezetimibe in oil, surfactant, and co-surfactant at close temperatures. The final mixture was vortexed for $24 \mathrm{hrs}$ to get a clear solution. Then all the mixtures are stored at room temperature for further use. Finally, the formulations were inspected for turbidity or phase separation indications before self-emulsification and particle size determination. Table 7 shows all different SEDDS formulations of Ezetimibe. Different ratios of oil and Smix were used to study various evaluation parameters of the optimized formulation. 
Application of Quality by Design Approach to Develop Novel Optimized Self-emulsifying Drug Delivery System of Ezetimibe for Treatment of Poorly Water-soluble Antilipidemic Drug to Enhance its Bioavailability by Using D-optimal Mixture Design

\begin{tabular}{|c|c|c|c|c|c|c|c|}
\hline $\begin{array}{l}\text { Formulation } \\
\text { Code }\end{array}$ & Visual Assessment & $\begin{array}{c}\text { Transmittance } \\
(\%) n-=3\end{array}$ & $\begin{array}{l}\% \text { Drug } \\
\text { content }\end{array}$ & $\begin{array}{c}\text { Emulsification } \\
\text { Time (sec) } n=3\end{array}$ & $\begin{array}{l}\text { Droplet } \\
\text { Size }\end{array}$ & PDI & Zeta Potential \\
\hline $\mathrm{F} 1$ & I & $98.06 \pm 0.14$ & 98.3 & $25 \pm 1$ & 78.25 & 0.222 & -12.7 \\
\hline F2 & I & $99.8 \pm 0.10$ & 99.98 & $21 \pm 1$ & 56.25 & 0.230 & -13.6 \\
\hline F3 & I & $99.1 \pm 0.10$ & 99.6 & $22 \pm 2$ & 75.35 & 0.381 & -11.1 \\
\hline $\mathrm{F} 4$ & I & $98.3 \pm 0.19$ & 99.8 & $24 \pm 1$ & 81.24 & 0.300 & -3.8 \\
\hline F5 & I & $99.5 \pm 0.32$ & 99.68 & $29 \pm 1$ & 59.25 & 0.171 & -12.41 \\
\hline F6 & I & $98.42 \pm 0.26$ & 99.5 & $25 \pm 2$ & 57.54 & 0.252 & -12.8 \\
\hline F7 & I & $97.72 \pm 0.71$ & 98.7 & $28 \pm 1$ & 75.23 & 0.290 & $-2.29 \mathrm{mv}$ \\
\hline F8 & I & $98.03 \pm 0.70$ & 98.87 & $24 \pm 1$ & 56.32 & 0.230 & $-7.46 \mathrm{mv}$ \\
\hline F9 & I & $98.53 \pm 0.83$ & 99.69 & $23 \pm 2$ & 65.25 & 0.310 & $-6.86 \mathrm{mv}$ \\
\hline F10 & I & $99.5 \pm 0.72$ & 99.3 & $25 \pm 1$ & 65.68 & 0.170 & $-9.74 \mathrm{mv}$ \\
\hline F11 & I & $98.72 \pm 0.71$ & 98.6 & $27 \pm 1$ & 57.54 & 0.280 & $-1.29 \mathrm{mv}$ \\
\hline F12 & I & $97.93 \pm 0.55$ & 99.03 & $26 \pm 1$ & 58.25 & 0.173 & $-0.144 \mathrm{mv}$ \\
\hline F13 & I & $97.19 \pm 0.90$ & 98.87 & $30 \pm 1$ & 78.84 & 0.300 & $-8.70 \mathrm{mv}$ \\
\hline F14 & I & $98.03 \pm 0.70$ & 98.5 & $26 \pm 2$ & 58.98 & 0.210 & $-7.86 \mathrm{mv}$ \\
\hline F15 & I & $97.23 \pm 0.83$ & 98.25 & $30 \pm 1$ & 89.58 & 0.290 & $-6.26 \mathrm{mv}$ \\
\hline F16 & I & $97.8 \pm 0.72$ & 98.4 & $29 \pm 1$ & 78.25 & 0.180 & $-7.74 \mathrm{mv}$ \\
\hline
\end{tabular}

Table 7: Evaluation of SEDDS F1 to F16 Formulation of d-optimal mixture design.

Effect of ezetimibe in phase diagram

They examine the impact of Ezetimibe on the self-Microemulsifying performance of SEDDS; $10 \mathrm{mg}$ of Ezetimibe was added to $1 \mathrm{ml}$ of a limit plan of SEDDS and checked for the formation of a clear solution. The effect of Ezetimibe on the phase diagram demonstrates in figure $4 \mathrm{D}-4 \mathrm{~F}$.

\section{Evaluation of SMDDS formulations}

\section{Self-emulsification time}

USP dissolution apparatus II use to evaluate the self-emulsification time of SEDDS. Then add one ml of every formulation in the $900 \mathrm{ml}$ of distilled water at $37 \pm 0.5^{\circ} \mathrm{C}$. Next, mild fomentation was given by a standard stainless steel dissolution paddle rotating at 50 pm. Emulsification time checked visually. Emulsification must complete within 1 minute $[1,19]$. The results of self-emulsification time exhibit in table 7.

\section{Visual assessment}

Take one $\mathrm{ml}$ of SEDDS diluted with $500 \mathrm{ml}$ of purified water at $37 \pm 0.5^{\circ} \mathrm{C}$. A stainless steel dissolution paddle rotating at $50 \mathrm{rpm}$ leads to gentle agitation. The time is taken (seconds) by every for- mulation to shape a clear homogenous framework noted in three sets. In light of the item's last debut, the emulsified batches were graded per the accompanying evaluating framework. Grade I: Quickly forming microemulsion which is explicit or slightly bluish in appearance within less than 1 minute. Grade II: Quickly forming, little less clear emulsion having a bluish-white appearance within 2 minutes. Grade III: milky white emulsion within 3 minutes. Grade IV: Tedious, grayish-white emulsion with an oily appearance that is slow to emulsification for more than 3 minutes. Grade V: Tedious, grayish-white emulsion with an oily appearance that is slow to emulsification for more than 3 minutes [20]. Result display in table 7.

\section{Transmission test}

The transmission test of optimized SEDDS formulations checked using a U.V. spectrophotometer (UV 1800, Shimadzu). First, the sample's percentage transmittance was measured at $650 \mathrm{~nm}$, with distilled water as the blank, and for each piece, triplicate assays were performed. Then calculate the percentage transmittance in $100 \mu \mathrm{L}$ of the SEDDS formulation was diluted with $100 \mathrm{ml}$ of distilled water $[3,21]$. Results of the transmission test are shown in table 7. 
Application of Quality by Design Approach to Develop Novel Optimized Self-emulsifying Drug Delivery System of Ezetimibe for Treatment of Poorly Water-soluble Antilipidemic Drug to Enhance its Bioavailability by Using D-optimal Mixture Design

Drug content

The Ezetimibe loaded- SEDDS formulation was dissolved in methanol. The solutions were filtered utilizing Whatman filter paper. The methanolic solution was estimated for the Ezetimibe content with the help of a U.V. spectrometer at $232 \mathrm{~nm}$ using a standard curve. Results of \% drug content display in table 7.

Robustness to dilution

This estimation was utilized to investigate the impact of dilution on SEDDS, with a particular ultimate objective to follow physiological dilution procedure after oral administration. The formulations were diluted in different proportions (1:50, 1:100, and 1:1000) with various diluents (distilled water, $0.1 \mathrm{~N} \mathrm{HCL}$, and acetate buffer (pH4.5)). The resultant emulsions were examined for any physical changes after storage for $24 \mathrm{hrs}$ [8]. Results of robustness to dilution are shown in table 8.

\begin{tabular}{|c|c|c|c|c|c|c|c|c|c|}
\hline \multirow{2}{*}{$\begin{array}{l}\text { Formulation } \\
\text { Code }\end{array}$} & \multicolumn{3}{|c|}{$2-4^{\circ} \mathrm{C}$} & \multicolumn{3}{|c|}{ Room Temperature } & \multirow{2}{*}{$\begin{array}{c}\begin{array}{c}\text { Centrifugation } \\
\text { Stability }\end{array} \\
\text { Phase separation }\end{array}$} & \multicolumn{2}{|c|}{ Robustness to dilution } \\
\hline & PS & $\mathbf{F}$ & PR & PS & $\mathbf{F}$ & PR & & Distilled water & 0.1N HCL \\
\hline F1 & $\mathrm{X}$ & $\mathrm{X}$ & $\mathrm{X}$ & $\mathrm{X}$ & $\mathrm{X}$ & $\mathrm{X}$ & $\mathrm{X}$ & $\sqrt{ }$ & $\sqrt{ }$ \\
\hline $\mathrm{F} 2$ & $\mathrm{X}$ & $\mathrm{X}$ & $\mathrm{X}$ & $\mathrm{X}$ & $\mathrm{X}$ & $\mathrm{X}$ & $\mathrm{X}$ & $\sqrt{ }$ & $\sqrt{ }$ \\
\hline F3 & $\mathrm{X}$ & $\mathrm{X}$ & $\mathrm{X}$ & $\mathrm{X}$ & $\mathrm{X}$ & $\mathrm{X}$ & $\mathrm{X}$ & $\sqrt{ }$ & $\sqrt{ }$ \\
\hline $\mathrm{F} 4$ & $\mathrm{X}$ & $\mathrm{X}$ & $\mathrm{X}$ & $\mathrm{X}$ & $\mathrm{X}$ & $\mathrm{X}$ & $\mathrm{X}$ & $\sqrt{ }$ & $\sqrt{ }$ \\
\hline F5 & $\mathrm{X}$ & $\mathrm{X}$ & $\mathrm{X}$ & $\mathrm{X}$ & $\mathrm{X}$ & $\mathrm{X}$ & $\mathrm{X}$ & $\sqrt{ }$ & $\sqrt{ }$ \\
\hline F6 & $\mathrm{X}$ & $\mathrm{X}$ & $X$ & $X$ & $X$ & $X$ & $X$ & $\sqrt{ }$ & $\sqrt{ }$ \\
\hline F7 & $\mathrm{X}$ & $\mathrm{X}$ & $\mathrm{X}$ & $X$ & $X$ & $X$ & $X$ & $\sqrt{ }$ & $\sqrt{ }$ \\
\hline F8 & $X$ & $X$ & $X$ & $\mathrm{X}$ & $X$ & $\mathrm{X}$ & $X$ & $\sqrt{ }$ & $\sqrt{ }$ \\
\hline F9 & $\mathrm{X}$ & $\mathrm{X}$ & $\mathrm{X}$ & $\mathrm{X}$ & $\mathrm{X}$ & $\mathrm{X}$ & $\mathrm{X}$ & $\sqrt{ }$ & $\sqrt{ }$ \\
\hline F10 & $\mathrm{X}$ & $X$ & $\mathrm{X}$ & $\mathrm{X}$ & $\mathrm{X}$ & $\mathrm{X}$ & $X$ & $\sqrt{ }$ & $\sqrt{ }$ \\
\hline F11 & $X$ & $X$ & $X$ & $X$ & $X$ & $X$ & $X$ & $\sqrt{ }$ & $\sqrt{ }$ \\
\hline F12 & $X$ & $\mathrm{X}$ & $X$ & $X$ & $X$ & $X$ & $X$ & $\sqrt{ }$ & $\sqrt{ }$ \\
\hline F13 & $X$ & $X$ & $X$ & $X$ & $\mathrm{X}$ & $X$ & $X$ & $\sqrt{ }$ & $\sqrt{ }$ \\
\hline F14 & $X$ & $X$ & $X$ & $\mathrm{X}$ & $X$ & $X$ & $X$ & $\sqrt{ }$ & $\sqrt{ }$ \\
\hline F15 & $\mathrm{X}$ & $\mathrm{X}$ & $\mathrm{X}$ & $X$ & $X$ & $\mathrm{X}$ & $X$ & $\sqrt{ }$ & $\sqrt{ }$ \\
\hline F16 & $X$ & $X$ & $X$ & $\mathrm{X}$ & $X$ & $X$ & $X$ & $\sqrt{ }$ & $\sqrt{ }$ \\
\hline
\end{tabular}

Table 8: Temperature stability study, Centrifugation Stability study and Robustness to dilution of F1 to F16 Formulations.

Footnote: $\mathrm{X}=$ Not seen $\sqrt{ }=$ seen $\mathrm{P}=$ Phase separation $\mathrm{F}=$ Flocculation $\mathrm{PR}=$ Precipitation

Where $\sqrt{ }$ is stable preparation

Droplet size determination and polydispersibility index (PDI)

Take one $\mathrm{ml}$ of SEDDS formulation and dilute with $100 \mathrm{ml}$ of water in a volumetric flask. Then the volumetric flask was inverted twice to ensure complete dispersion of the formulation. After confirming the total distribution of formulation, the droplet size of the resultant microemulsion was estimated using photon correlation spectroscopy that analyzes the fluctuation in light scattering due to the Brownian motion of the globule as a purpose of time utilizing a Zetasizer Nano Series. Light scattering was examined at $25^{\circ} \mathrm{C}$ at $90^{\circ} \mathrm{C}$ angles [22-25]. Results show in table 7.

\section{Zeta potential determination}

The stability of the emulsion is straightforwardly recognized with the actual surface charge. One ml of SEDDS was diluted 100 
Application of Quality by Design Approach to Develop Novel Optimized Self-emulsifying Drug Delivery System of Ezetimibe for Treatment of Poorly Water-soluble Antilipidemic Drug to Enhance its Bioavailability by Using D-optimal Mixture Design

times with distilled water in a beaker with continuous stirring on a magnetic stirrer. The Zeta-potential of the SEDDS formulations was determined using a Malvern Zetasizer [26,27]. Results present in table 7.

Optimization of Ezetimibe-loaded SEDDS formulations using d-optimal mixture design

The d-optimal mixture design was used to optimize the composition of the SEDDS formulation. The experiment was designed using the three components as independent variables [3]. Based on the solubility study and pseudo-ternary phase diagram, concentrations of Capmul PG-8 (oil; X1), Kolliphor RH 40 (surfactant; $\mathrm{X} 2$ ), and Transcutol $\mathrm{P}$ (cosurfactant; X3) were set within ranges of $15 \%-20 \%$, 30\%-50\%, and 30\%-50\%, correspondingly. For any test, the focuses of X1, X2, and X3 added up to 100\%. Percentage drug content (Y1), Percentage Drug Release in 20 minutes (Y2), Emulsification time in second (Y3), and Mean droplet size (Y4) were evaluated to determine the optimal SEDDS formulation with excellent physiochemical characteristics. We used JMP@ Trail 16 Version (SAS, New Jersey, USA) to develop and evaluate the trial plan. The base design allowed 16 trials to fit the best model and estimate experimental error responses (Y1Y2, Y3, and Y4) shown in table 5 and 6 . The CPP was not calculated in the plan thought due to their insignificant impact on the reactions as shown by the Risk Estimated Matrix (REM). The different batches (Table 6) got according to the plan are exposed to the portrayal. Coherent validation of different $\operatorname{risk}(\mathrm{s})$ for each of the material attributes and process factors resultant to the individual CQAs display in table 4.

\section{Transmission electron microscopy (TEM)}

The morphology of the emulsion drop for the optimized Ezetimibe-loaded SEDDS formulation was noticed utilizing a TEM (JEM 1010, JEOL Ltd, Tokyo, Japan) with a speed increase voltage of $80 \mathrm{kV}$. The enhanced SEDDS formulation was diluted with water $(1: 1,000)$. One drop of the example was straightforwardly stored on a copper network and dried at $25^{\circ} \mathrm{C}$.

\section{In vitro dissolution studies}

In-vitro release investigations of SEDDS were carried out utilizing USP dissolution apparatus type-II with rotating paddle at 50 rpm and maintained at $37 \pm 0.5^{\circ} \mathrm{C}$ temperature in dissolution media pH 1.2 buffers $(900 \mathrm{ml})$ to examine drug discharge from SEDDS. The Ezetimibe loaded-SEDDS formulation and pure drug were filled in a hard gelatin capsule and introduced into the dissolution medium. At predetermined time intervals of $5 \mathrm{~min}$ to $1 \mathrm{hr}, 10 \mathrm{ml}$ of the samples were drawn and filtered through $0.45 \mu \mathrm{m}$ Whatman filter paper. The fresh dissolution medium was simultaneously replaced in the apparatus to keep the steady volume. The filtered solution was analyzed using a U.V. spectrophotometer at $232 \mathrm{~nm}$ to check the amount of drug release in dissolution media. All estimations were done in triplicate $[28,29]$. The graph was plotted against drug release $\mathrm{v} / \mathrm{s}$ time to investigate formulations and pure drugs, as demonstrated in figure 7.

\section{Thermodynamic stability study}

All SEDDS formulations of Ezetimibe were disclosed to the heating-cooling cycle and centrifugation to investigate thermodynamic stability:

- Heating cooling cycle: The SEDDS formulation is put in a refrigerator temperature at 2 to $4^{\circ} \mathrm{C}$ and room temperature with storage at each temperature less than $48 \mathrm{hrs}$. Each one of those arrangements, which are steady at this temperature, was further investigated through centrifugation.

- Centrifugation: The formulations, which were cleared the heating-cooling cycle, were centrifuged at $3500 \mathrm{rpm}$ for 30 min. Table 8 demonstrate the results of the thermodynamics stability study.

- $\quad$ Freeze-thaw cycle: we use three freeze-thaw cycles at $-21^{\circ} \mathrm{C}$ and $+25^{\circ} \mathrm{C}$ with storage at every temperature for not less than 48 hours for the formulations.

\section{Result and Discussion}

\section{Risk assessment}

The dosage form development under the QbD framework involves evaluating material and process attributes, significantly influencing product quality. Through the fishbone chart (Figure 1), the potential variables influencing the product CQAs were recognized. Regarding SEDDS readiness, the material ascribes like oil, surfactant, and cosurfactant/cosolvent significantly contribute to product responses than the process attributes because the preparation method is simple. Hence, we use the operation characteristics involved in the SEDDS preparation like stirring time, temperature, and stirring speed as the least preferred because of their minimal contribution towards the product inconstancy. Thus, the 
threat related to measuring boundaries is assessed as low (Table 3). The practical headway of SEDDS formulations depends upon the proper decision of excipients with their general degree in the definition formulation [9,13-15].

\section{Preformulation study}

Screening of vehicles: (Oil, Surfactant, Co- surfactants)

Solubility study was performed to identify suitable oil phase, surfactant, and co-surfactant to develop ezetimibe SEDDS. Maximum solubilizing capacities of components (oil, surfactant, and co-surfactant) are necessary to achieved optimum drug loading [30,31].

\section{Selection of oil}

The oil is one of the critical excipients within the SEDDS formulation due to the fact it could solubilize commercialized amounts of the lipophilic drug or facilitate self-emulsification. Moreover, mainly because it can enhance the segment of lipophilic drug transported through the intestinal lymphatic system. So, that absorption from the G.I. tract relaying on the molecular nature of the triglyceride. Therefore, long and medium-chain triglycerides oils with different degrees of saturation have been used to formulate a selfmicro emulsifying drug delivery system.

The solubility study described in the section the solubility of Ezetimibe in different oil show in figure 3A, Screening of appropriate oil is the primary requirement of SEDDS development. Therefore, solubility studies were aimed to identify suitable oil having maximal solubilizing potential for the development of SEDDS. Capmul PG-8 was found to solubilize the maximum amount of Ezetimibe ( $99.73 \mathrm{mg} / \mathrm{ml}$ ) among the selected oil. However, the other oils exhibited different solubilizing capacities, reported in table 7 and figure 3. Hence, (Capmul PG-8) was preferred for further study.

\section{Selection of surfactants}

The surfactant concerned with the SEDDS formulation has a high HLB value around 8.6 to 16.7 and hydrophilicity of surfactant, which cause instant development of oil in water droplet and quick dispersion of formulation aqueous media like gastrointestinal fluid. The drug dispersed within the SEDDS formulation would remain solubilized for a long time at the absorption site, thus preventing precipitation of drug compounds inside G.I. lumen surfactant were absorbed in the interface because of its amphiphilic nature, and they can dissolve a high amount of lipophilic drug in the oily phase.

According to the solubility method described in the selection of surfactant, the solubility of Ezetimibe in different surfactants represents in figure 3B. Within the solubility data of Ezetimibe in various surfactants, the highest solubility of the drug was found in Kolliphor RH40 (57.21mg/ml) and Acrysol K160 (52.21mg/ $\mathrm{ml}$ ). The oily phase Capmul PG-8 showed the highest emulsification performance with Kolliphor RH40 for the homogenous emulsion formulation, and additionally, Acrysol K160 produced better transparency in the formulation. On the other hand, Capmul PG-8 showed poor emulsification properties with other surfactants. Therefore, Kolliphor RH 40 surfactant was selected for SEDDS formulation. A proper amount of low and high HLB value surfactants were required to form the steady microemulsion in preparing a self-emulsified formulation. Therefore, Kolliphor RH 40 with an average HLB value of 15 and Transcutol P with an HLB value of 4 were utilized. Campus PG-8 is entrapping in the surfactant with more HLB value, enhancing the emulsification process upon dilution with an aqueous medium. In addition, these excipients provide better stability to the emulsion.

\section{Selection of co-surfactants}

The co-surfactant use to diminish the oil-water interface fluidizes the interfacial film's hydrocarbon area and licenses the spontaneous development of microemulsion. Therefore, the selection of a cosurfactant is critical to forming microemulsion formation and solubilization in microemulsion. According to the solubility method described in the selection of the co-surfactant, the solubility of Ezetimibe in different co-surfactant describe in figure 3C. Among the solubility information of Ezetimibe in distinctive co-surfactant, most of the drug dissolved in Transcutol P (135.2mg/ml), which indicates $99.40 \%$ transparency. Accordingly, Transcutol P chose as a cosurfactant in the SEDDS formulation. Furthermore, the investigation recognizes the ability of various co-surfactants to enhance the microemulsion of selected surfactants. Therefore, Increase the spontaneity of the microemulsion formulation increased by increasing the co-surfactant.

Oil, surfactant, and co-surfactant are constructing excipients of the self-Microemulsifying drug delivery system. The drug needs to be soluble in all three excipients in the mixture. Therefore, the 
Application of Quality by Design Approach to Develop Novel Optimized Self-emulsifying Drug Delivery System of Ezetimibe for Treatment of Poorly Water-soluble Antilipidemic Drug to Enhance its Bioavailability by Using D-optimal Mixture Design

solubility of the drug needs to be suitable for the choice of oil, surfactant, and co-surfactant. Moreover, the solubility of the drug is likewise crucial to dictate the dose of SEDDS. Hence, SEDDS need to comprise oil, surfactant, and co-surfactant that accommodate the amount of the drug. Another element, which might be affected due to solubility, is the partitioning effect. If the drug isn't always appropriate in the mixture, it will diffuse towards the water when developing microemulsion. Each of those facts, choice of excipients is a crucial factor for successful formulation. The solubility of oil data (Figure 3A) indicates that Ezetimibe has better solubility in synthetic oil than vegetable oil.so; Capmul PG-8 was selected as the oil phase. Kolliphor RH40 acts as a surfactant because of its extreme HLB value. Therefore it showed good solubility of Ezetimibe in the oil phase. The third element of SEDDS, i.e., the Co-surfactant (Transcutol P), helps surfactant stabilize the system.

\section{Drug-excipient compatibility study}

FT-IR of formulation and the drug were carried out to investigate any drug-excipient interactions using $\mathrm{KBr}$ disc along with scanning range of 4000-400 $\mathrm{cm}^{-1}$ and resolution $1 \mathrm{~cm}^{-1}$. The I.R. spectrum of the drug was in contrast with the standard I.R. spectra of Ezetimibe. The FTIR spectra of ezetimibe confirmed peaks at 3411.84-3235.30 $\mathrm{cm}^{-1}$ (O-H, Alcohol/Phenol), 3085.75-3015.34 $\mathrm{cm}^{-1}$ (C-H, Aromatic), $1684.28 \mathrm{~cm}^{-1}$ (N- C=O Amide), and 1097.31 $\mathrm{cm}^{-1}$ (C-F bending). These peaks might be considered as ideal peaks of Ezetimibe and had been now no longer affected and prominently noticed in I.R. spectra of Ezetimibe together with oil, surfactant, and co-surfactant. So, from the comparison, it was concluded that the drug and excipients were suitable for each other. The FTIR spectra of pure Ezetimibe drug and Ezetimibe formulation's overlapping spectra were proven in figure $2 \mathrm{~A}$ and $2 \mathrm{~B}$. Comparison of vibration Frequency of FTIR spectra of Ezetimibe and Ezetimibe formulation indicates there might be no important distinction in characteristics peak at a wavenumber of the drug within the presence of excipients. It indicates that formulations became well suited with excipients.

\section{Pseudo-ternary phase diagram}

The self-micro emulsifying system produces oil in water emulsion with gentle agitation into the aqueous medium. A surfactant or co-surfactant preferentially adsorbed on the interface, lowering the interfacial energy and offering a mechanical barrier to coalescence. The reductions of the free energy required for the micro- emulsion formulation concurrently improve the microemulsion formulation's thermodynamic balance. Therefore, the choice of oil, surfactant, co-surfactant, and the combination ratio of oils to Smix play a vital role in the microemulsion formulation.

Based on the results of preliminary studies, a ternary phase diagram was constructed to study the relationship between the phase behavior and composition of SEDDS. It also helps to determine the concentration range of components for the formulation of a microemulsion. It had used a combination of surfactant (Smix) with high and low HLB values in the present work. Campus PG-8 has less HLB value, and Kolliphor RH 40 having a high HLB value. A combination of low and high HLB surfactant leads to more rapid dispersion and finer emulsion droplets size in addition to the aqueous segment. Campus PG-8 and Kolliphor RH 40 withinside the proportion of 1:1 indicated higher microemulsion region and rapid emulsification compared with 2:1 and 1:2 Smix selected for formulation development.

Different ratios, as according to figure 4A-4C were considered for optimization of the proportion of oil, surfactant, and cosurfactant for microemulsion. As per figure $4 \mathrm{~A}$, a 1:1 ratio was used. The data showed that up to $1: 9$ to $4: 6$ parts of Oil to Smix ratio gave a clear solution when it was titrated with 100 parts of the water because Smix parts are higher than oil parts. However, after 4:6, parts of oil to Smix produced turbidity in the solution, indicating that SEDDS was unstable on dilution. Therefore, as above figure 4B, SEDDS were prepared by using a 2:1 Smix ratio. The data found that from 1:9 to 3.5:6.5, parts of oil to Smix give clear solution when it was titrated up to 100 parts of water because oil parts are less than Smix parts. Therefore, surfactant reduces the interfacial tension between oil and water lead to a clear solution. From figure 4C, a 1:2 ratio was used. The data found that up to $1: 9$ to $3: 7$, parts of the oil to Smix ratio gave a clear solution when it was titrated up to 100 parts of water. The nature of microemulsion formed in the aqueous medium depends on the concentration of Smix (a mixture of Kolliphor RH 40 and Transcutol P) in the formulation. Figure 3A$3 \mathrm{C}$ observed that as the concentration of Smix was increased with decreasing concentration of oil, it improved the clarity of the selfmicro emulsifying system. In addition, the surfactant reduced the oil-water interface, which made rapid dispersion of SEDDS in an aqueous medium and reduced in particle size when diluted with water. 
Application of Quality by Design Approach to Develop Novel Optimized Self-emulsifying Drug Delivery System of Ezetimibe for Treatment of Poorly Water-soluble Antilipidemic Drug to Enhance its Bioavailability by Using D-optimal Mixture Design

Effect of ezetimibe in the phase diagram

As per the phase diagram (Figure 4D-4F), different ratios considered optimizing the proportion of oil, surfactant, and co-surfactant for the Ezetimibe microemulsion system. The microemulsion was prepared using Capmul PG-8, Kolliphor RH 40, and Transcutol $\mathrm{P}$ as the main three constituents of the formulation. A formulation was designed with a S/Co-surfactant ratio of 1:1, 2:1, 1:2. When the amount of oil is less than $10 \%$, the water content is $90 \%$. At this point, microemulsion can be diluted to infinite. Which fulfilled the requirements of SEDDS, and also droplet size is less than $100 \mathrm{~nm}$.

The hydrophobic drug was entrapped in the SEDDS, which affects the self-emulsifying performance. Also, precipitation of the drug may occur in the gut during the dilution by body fluids due to the loss of solvent capacity in a co-surfactant present in the microemulsifying drug delivery system. In the present study, a reduction in the efficient self-emulsifying domain was observed by incorporation with Ezetimibe.

Optimization of Ezetimibe-loaded SEDDS using desirability function

Three highlights are required for streamlining: cutoff esteem (upper or lower), dispensed objective value, and information showing whether every response should be better or limited. When a streamlining interaction is combined with the increase, an incentive for the lower bound is figure 5B. Impacts of independent factors on the reaction factors, Y1, Y2, Y3, Y4. Three-dimensional response surface plot for the impacts of the independent factors. Contour plots for the impacts of $\mathrm{X} 1$ and $\mathrm{X} 2$ on the reactions. (Y1, percentage drug content; $Y 2$, the rate of drug discharge in $20 \mathrm{~min}$; Y3 emulsification time in second, and Y4, the drop size in $\mathrm{nm}$ ).

Conversely, when a response variable is limited, an incentive for the upper bound is required. Y1 was set to be amplified, and an objective worth and the cutoff worth of 95 and100\%, individually, were chosen. The lower bound of $95 \%$ was chosen dependent on the most reduced level of medication content in the SEDDS formulation. Y2 was set to be boosted, and an objective worth and the cutoff worth of 100 and $80 \%$, individually, were chosen. The lower bound of $80 \%$ was chosen dependent on the most reduced medication delivered from SEDDS formulation in $20 \mathrm{~min}$ in $\mathrm{pH} 1.2$ medium. The objective worth of $100 \%$ was chosen depending on the most noteworthy medication discharge quickly (Table 5). A little drop size permits better medication absorption since it gives an expanded surface region to the retention and permits quicker medication discharge. Dissolution is a rate-restricting advance for the oral absorption of inadequately water-dissolvable medications, particularly drugs having a place with class II of the Biopharmaceutics Classification System, including Ezetimibe. Further, to expand proficiency and decline work escalation, a disintegration time of 20 minutes was embraced to decide the connection between independent factors and dissolution. Y3 was set to be a target match and an objective worth and lower bound of 20 seconds and 60 seconds individually, were chosen. The lower bound of 20 seconds was picked dependent on the miscibility in the water and the structure of a steady emulsion (Table 5). Y4 was set to be limited because the more modest the globule sizes of SEDDS formulation, the better the G.I. assimilation. A few examinations on SEDDS and microemulsions proposed that the ideal distance across a steady microemulsion ought to be $<200$. In our exploratory run of blend plan, the base trial worth of drop size was $56.25 \mathrm{~nm}$ (Table 7). In this manner, both the objective worth and upper bound were 50 and $100 \mathrm{~nm}$, individually, for the enhancement. All reaches for the reaction factors were among the upsides of our trial run of a combination plan. The independent factors were improved for the reaction esteems by utilizing the attractive quality capacity. Figure $5 \mathrm{~A}$ addresses the contour and surface plot for the impacts of the three free factors on all response factors.

\begin{tabular}{|l|c|c|c|c|}
\hline Parameters & Y1 & Y2 & Y3 & Y4 \\
\hline R Square & 0.952 & 0.995 & 0.998 & 0.990 \\
\hline R Square Adj & 0.958 & 0.975 & 0.998 & 0.996 \\
\hline Root Mean Square Error & 1.16 & 8.6 & 0.03 & 16.6 \\
\hline Mean of response & 99.06 & 69.08 & 25.56 & 63.92 \\
\hline
\end{tabular}

Table 9: Summary of fit.

The Optimized formulation proportions of Ezetimibe-loaded SEDDS of X1, X2, and X3 were 18\%, 40.97\%, and 40.95\%, individually. An attractive quality capacity worth of 0.000196 upheld these qualities (Figure 5A). The anticipated and noticed upsides of Y1, Y2, Y3, and Y4 for the improved Ezetimibe-loaded SEDDS formulations appear in table 10. Upsides of expectation blunders were determined to assess the dependability and precision. The model approval was done through the ternary mixture profiler. The ternary blend profiler gives the ideal space in the ternary graph (Figure 
Application of Quality by Design Approach to Develop Novel Optimized Self-emulsifying Drug Delivery System of Ezetimibe for Treatment of Poorly Water-soluble Antilipidemic Drug to Enhance its Bioavailability by Using D-optimal Mixture Design

5C). The distinctive proportion of oil, surfactant, and cosurfactant inside the perfect area doesn't influence the dependent factors (Responses) of the SEDDS definition. Albeit the expectation blunder of Y1 and Y2 was moderately high, its variable achieved a size that appeared to be good thinking about the exploratory scope of the SEDDS plan. The forecast mistakes of both Y3 and Y4 were a little greater than usual. Consequently, these outcomes showed that the D-Optimal mixture design technique utilized for optimizing Ezetimibe -stacked SEDDS in this investigation was dependable and exact. For the level of medication delivered in $20 \mathrm{~min}$ (Y2), the improved Ezetimibe-stacked SEDDS showed Y3 worth of 99.4\%. Also, it showed attractive upsides of percentage drug content (99.98\%) , $\%$ drug release in 20 minute (80.25\%) emulsification time $(\mathrm{Y} 3=$ 21 second). And bead size ( $\mathrm{Y} 4=56.25 \mathrm{~nm}$ ). Along these lines, F2 optimize formulation was exposed to promote in vitro dissolution study. The model was analyzed in JMP software. After Analysis, actual by predicted profile plot and contour profiler for globule size and $\%$ medicament release were obtained as shown in figure $5 \mathrm{~B}$.
The summary of fit and parameter estimates was also obtained, shown in table 9 and table 11. In the meantime, the transmission electron microscopy investigation for the optimized formulation SEDDS showed that the emulsion drops were round in the nanometer range, as demonstrated in figure 8.

\begin{tabular}{|l|c|c|c|}
\hline \multirow{2}{*}{ Responses } & \multicolumn{3}{|c|}{ OF-SEDDS } \\
\cline { 2 - 4 } & $\begin{array}{c}\text { Predicted } \\
\text { value }\end{array}$ & $\begin{array}{c}\text { Experimen- } \\
\text { tal value }\end{array}$ & $\begin{array}{c}\text { \% predic- } \\
\text { tion error }\end{array}$ \\
\hline \% Drug content (Y1) & 98.52 & 99.98 & 1.48 \\
\hline $\begin{array}{l}\text { \% Drug release in 20 } \\
\text { Minutes (Y2) }\end{array}$ & 64.75 & 80.25 & 20.93 \\
\hline $\begin{array}{l}\text { Emulsification time } \\
\text { (sec) (Y3) }\end{array}$ & 27.2 & 21 & -22.42 \\
\hline Droplet size (nm) (Y4) & 66.88 & 56.45 & -15.59 \\
\hline
\end{tabular}

Table 10: Experimental and predicted values for the optimized SEDDS formulation.

Difference $\%=[($ Experimental value - Predicted value $) /($ Predicted value)] x 100 .

\begin{tabular}{|c|c|c|c|c|c|}
\hline \multicolumn{6}{|c|}{ Parameter Estimate for Response Y1 } \\
\hline Components & Coefficient Estimate & Std Error & DFDen & t Ratio & p-value \\
\hline A (Capmul PG-8-0.15)/0.25 & 490.70 & 808.3 & 5.707 & 0.051 & 0.63 \\
\hline B (Kolliphor RH 40-0.3)/0.25 & 102.43 & 1.927 & 1.389 & 53.34 & $0.0029^{*}$ \\
\hline C (Transcutol P-0.3)/0.25 & 102.34 & 2.56 & 5.08 & 39.97 & $<.0001^{*}$ \\
\hline $\mathrm{A}^{*} \mathrm{~B}$ & -526.37 & 1354.38 & 5.714 & -0.39 & 0.7116 \\
\hline $\mathrm{A}^{*} \mathrm{C}$ & -530.15 & 1337.79 & 5.68 & -0.40 & 0.7063 \\
\hline $\mathrm{B}^{*} \mathrm{C}$ & -15.25 & 7.52 & 5.661 & -2.03 & 0.0921 \\
\hline $\mathrm{A}^{*} \mathrm{~B}^{*} \mathrm{C}$ & 479.70 & 1135.69 & 5.69 & 0.42 & 0.6882 \\
\hline$A^{*} B^{*}(A-B)$ & -202.36 & 575.95 & 5.723 & -0.35 & 0.7379 \\
\hline$A^{*} C^{*}(A-C)$ & -203.09 & 546.04 & 5.528 & -0.37 & 0.7238 \\
\hline $\mathrm{B}^{*} \mathrm{C}^{*}(\mathrm{~B}-\mathrm{C})$ & 7.661 & 10.04 & 5.182 & 0.76 & 0.4790 \\
\hline \multicolumn{6}{|c|}{ Parameter Estimate for Response Y2 } \\
\hline A (Capmul PG-8-0.15)/0.25 & 1900.34 & 31148.83 & 5.802 & 0.06 & 0.9534 \\
\hline B (Kolliphor RH 40-0.3)/0.25 & 90.95 & 27.19 & 4.416 & 3.34 & $0.0247^{*}$ \\
\hline C (Transcutol P-0.3)/0.25 & 63.44 & 20.70 & 5.075 & 3.06 & $0.0274^{*}$ \\
\hline $\mathrm{A}^{*} \mathrm{~B}$ & -7811.9 & 13911.74 & 4.787 & -0.56 & 0.5997 \\
\hline $\mathrm{A}^{*} \mathrm{C}$ & -7171.1 & 13788.65 & 4.844 & -0.52 & 0.6259 \\
\hline $\mathrm{B}^{*} \mathrm{C}$ & -42.34 & 77.16 & 5.987 & -0.55 & 0.6030 \\
\hline $\mathrm{A}^{*} \mathrm{~B} * \mathrm{C}$ & 5992.64 & 11681.21 & 4.78 & 0.51 & 0.6308 \\
\hline$A^{*} B^{*}(A-B)$ & -3416.48 & 5906.61 & 4.745 & -0.58 & 0.5893 \\
\hline
\end{tabular}

Citation: Mukesh S Patel., et al. "Application of Quality by Design Approach to Develop Novel Optimized Self-emulsifying Drug Delivery System of Ezetimibe for Treatment of Poorly Water-soluble Antilipidemic Drug to Enhance its Bioavailability by Using D-optimal Mixture Design". Acta Scientific Pharmaceutical Sciences 5.8 (2021): 20-42. 
Application of Quality by Design Approach to Develop Novel Optimized Self-emulsifying Drug Delivery System of Ezetimibe for Treatment of Poorly Water-soluble Antilipidemic Drug to Enhance its Bioavailability by Using D-optimal Mixture Design

\begin{tabular}{|c|c|c|c|c|c|}
\hline $\mathrm{A}^{*} \mathrm{C} *(\mathrm{~A}-\mathrm{C})$ & -2645.1 & 5691.58 & 4.969 & -0.46 & 0.6618 \\
\hline $\mathrm{B}^{*} \mathrm{C}^{*}(\mathrm{~B}-\mathrm{C})$ & -107.83 & 106.96 & 5.995 & -1.01 & 0.3523 \\
\hline \multicolumn{6}{|c|}{ Parameter Estimate for Response Y3 } \\
\hline A (Capmul PG-8-0.15)/0.25 & 1900.3478 & 31148.83 & 5.802 & 0.06 & 0.9534 \\
\hline B (Kolliphor RH 40-0.3)/0.25 & 27.272403 & 41.28062 & 3.222 & 0.66 & 0.5530 \\
\hline C (Transcutol P-0.3)/0.25 & 18.942631 & 105.1214 & 5.573 & 0.18 & 0.8634 \\
\hline $\mathrm{A}^{*} \mathrm{~B}$ & -3224.652 & 51002.37 & 5.788 & -0.06 & 0.9517 \\
\hline $\mathrm{A}^{*} \mathrm{C}$ & -2997.076 & 54762.99 & 5.844 & -0.05 & 0.9582 \\
\hline $\mathrm{B} * \mathrm{C}$ & 7.8657411 & 327.9032 & 5.565 & 0.02 & 0.9817 \\
\hline $\mathrm{A}^{*} \mathrm{~B}^{*} \mathrm{C}$ & 2680.9995 & 44120.79 & 5.822 & 0.06 & 0.9536 \\
\hline$A^{*} B^{*}(A-B)$ & -1422.454 & 19608.58 & 5.695 & -0.07 & 0.9447 \\
\hline $\mathrm{A}^{*} \mathrm{C}^{*}(\mathrm{~A}-\mathrm{C})$ & -1154.253 & 27096.04 & 5.93 & -0.04 & 0.9674 \\
\hline $\mathrm{B}^{*} \mathrm{C} *(\mathrm{~B}-\mathrm{C})$ & -16.99015 & 85.29569 & 0.141 & -0.20 & 0.9350 \\
\hline \multicolumn{6}{|c|}{ Parameter Estimate for Response Y4 } \\
\hline A (Capmul PG-8-0.15)/0.25 & 15403.815403 .566 & 16073.96 & 1.48 & 0.96 & 0.4678 \\
\hline B (Kolliphor RH 40-0.3)/0.25 & 93.51104 & 74.41515 & 2.104 & 1.26 & 0.3304 \\
\hline C (Transcutol P-0.3)/0.25 & -50.08988 & 61.35265 & 2.594 & -0.82 & 0.4825 \\
\hline$A^{*} B$ & -24916.68 & 26734.35 & 1.462 & -0.93 & 0.4791 \\
\hline $\mathrm{A}^{*} \mathrm{C}$ & -25395.87 & 26757.59 & 1.505 & -0.95 & 0.4696 \\
\hline $\mathrm{B}^{*} \mathrm{C}$ & 177.54653 & 233.7684 & 2.458 & 0.76 & 0.5137 \\
\hline $\mathrm{A}^{*} \mathrm{~B} * \mathrm{C}$ & 20277.438 & 22333.29 & 1.462 & 0.91 & 0.4884 \\
\hline$A^{*} B^{*}(A-B)$ & -9336.423 & 11237.48 & 1.417 & -0.83 & 0.5226 \\
\hline $\mathrm{A}^{*} \mathrm{C}^{*}(\mathrm{~A}-\mathrm{C})$ & -11415.34 & 11182.32 & 1.602 & -1.02 & 0.4366 \\
\hline $\mathrm{B}^{*} \mathrm{C} *(\mathrm{~B}-\mathrm{C})$ & -239.8532 & 286.8052 & 1.945 & -0.84 & 0.4931 \\
\hline
\end{tabular}

Table 11: Parameter estimate.

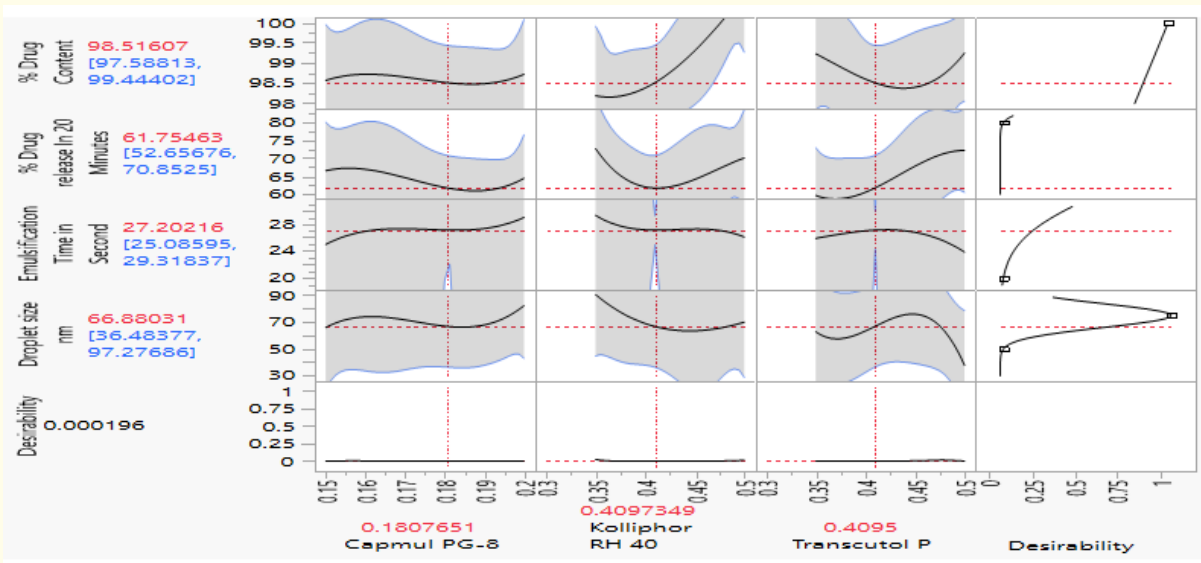

Figure 5A: Prediction profiler for multiple responses optimization SEDDS Formulation. 


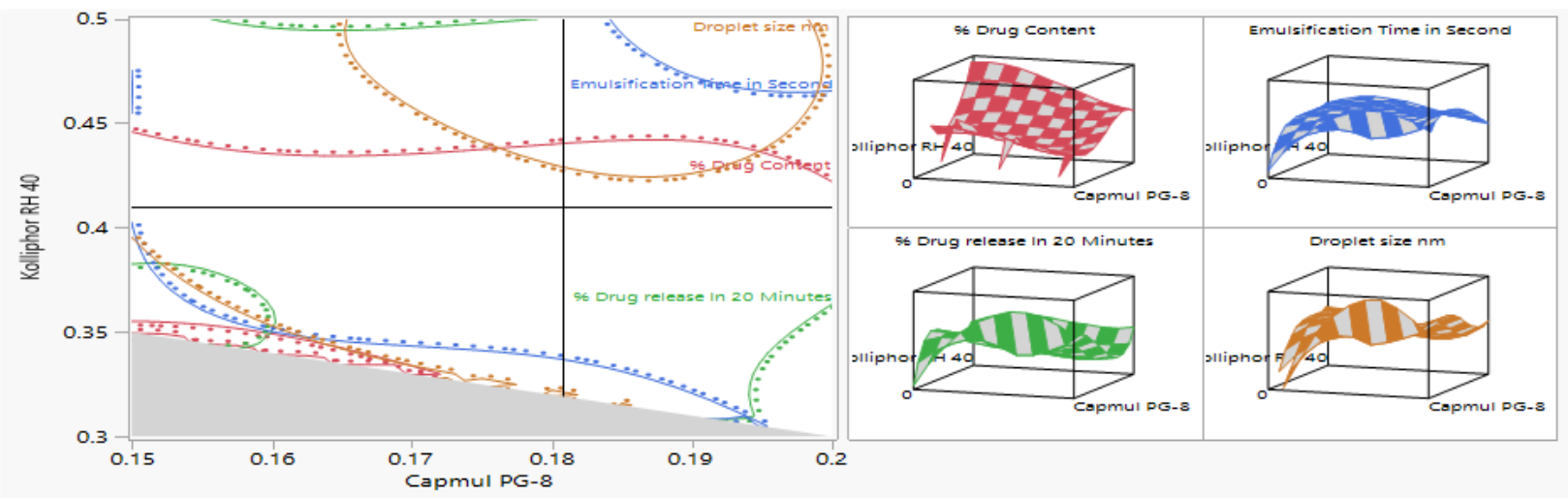

Figure 5B: Contour and surface plots exhibiting the impact of formulation components on the responses.

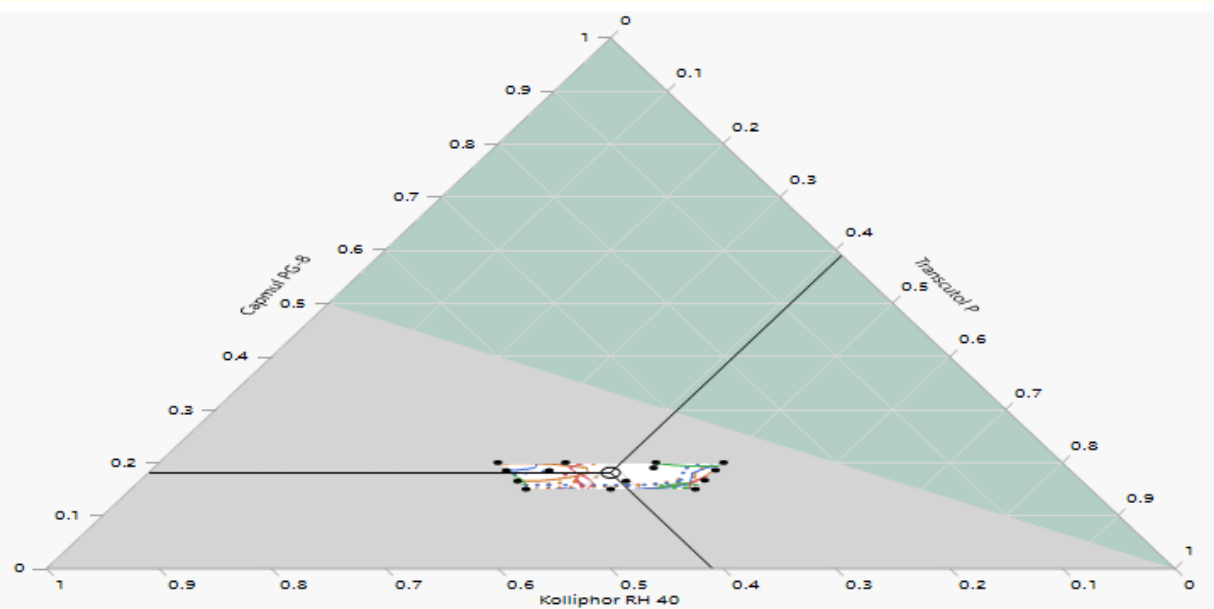

Figure 5c: Ternary Mixer profiler displaying the impact of formulation components on the responses.

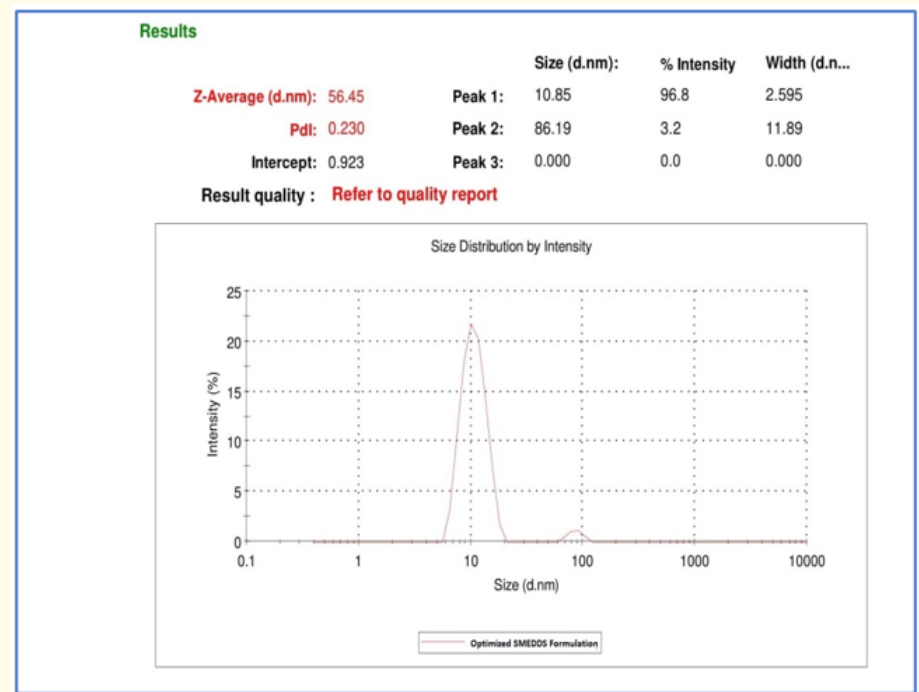

Figure 6A: Results of droplet size and PDI for the Optimization SEDDS Formulation. 


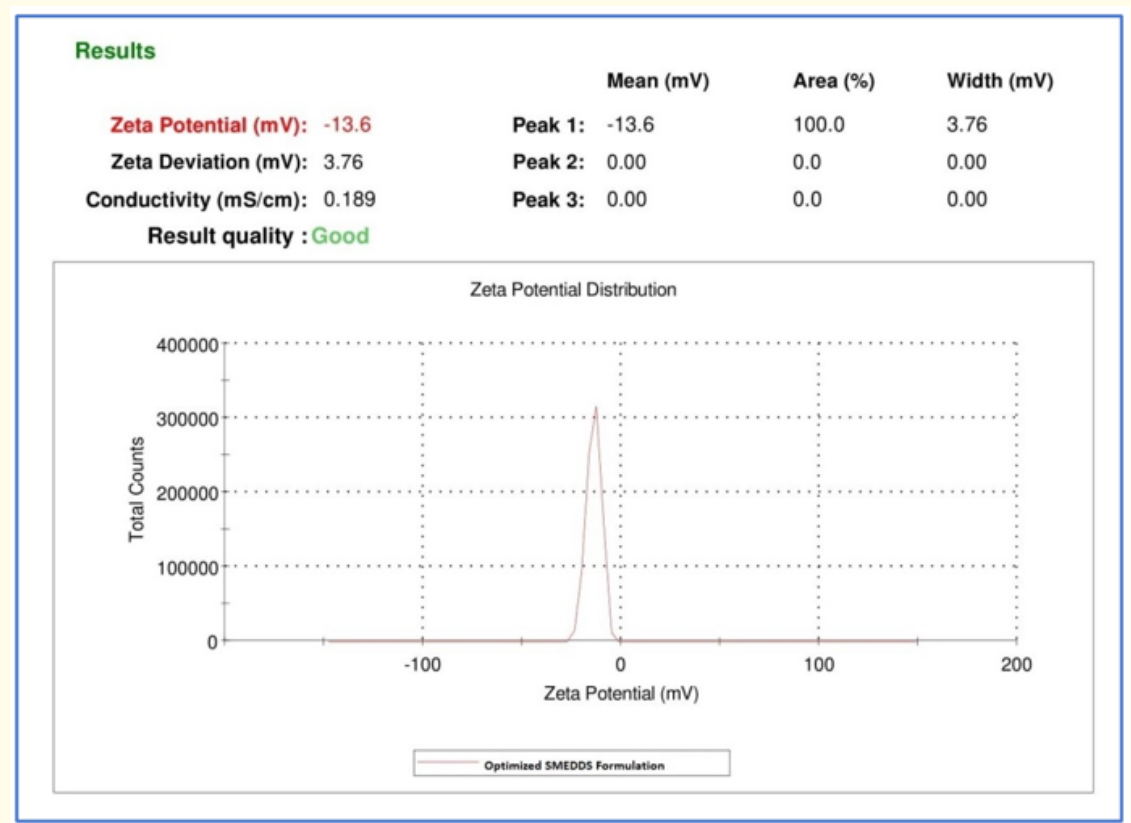

Figure 6B: Results of droplet size and PDI for the Optimization SEDDS Formulation.
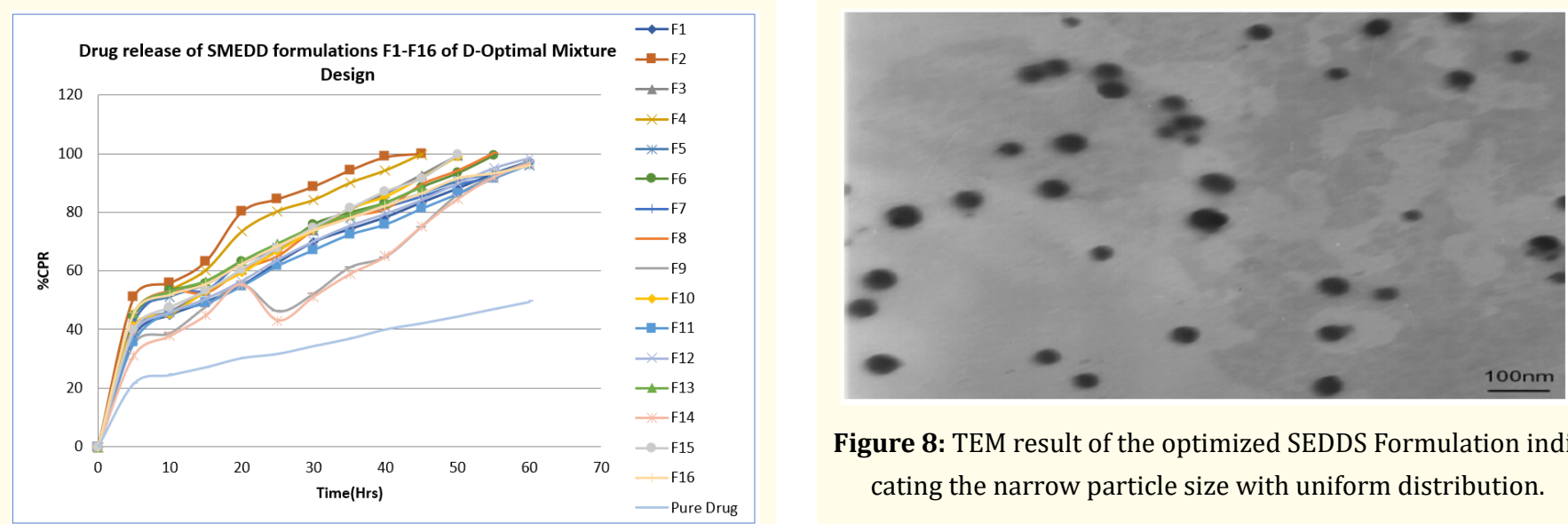

Figure 8: TEM result of the optimized SEDDS Formulation indicating the narrow particle size with uniform distribution.

Figure 7: Dissolution profile of SMEDDS formulations F1-F16 and pure drug. 
JMP software was used to fit all responses to linear models. The sequential P-values for Y1, Y2, Y3, and Y4 were 0.001810 .00189 , 0.0185 , and 0.0195 , respectively. A sequential P-value of less than 0.05 indicates that the model terms are significant. ANOVA resulted in 12.65 F value with Prob > F value of 0.00181 for $\mathrm{Y} 1$ and $45.00 \mathrm{~F}$ value for Y2 with Prob > F value of 0.0189, 12.89 F value with Prob $>$ F value of 0.00185 for $\mathrm{Y} 3$ and $35.00 \mathrm{~F}$ value for Y2 with Prob > $\mathrm{F}$ value of $0.0195, \mathrm{R}^{2}$ values for the responses $\mathrm{Y} 1, \mathrm{Y} 2, \mathrm{Y} 3$, and $\mathrm{Y} 4$ were approximately 0.952. 0.995, 0.998 and 0.990 respectively. The adjusted $R^{2}$ values for the responses $Y 1, Y 2, Y 3$, and Y4 were approximately $0.958,0.975,0.998$, and 0.996 , respectively. For a good model, similar values of $\mathrm{R}^{2}$ and adjusted $\mathrm{R}^{2}$ are desirable.

\section{Evaluation of SEDDS formulation}

Visual assessment

The tendency to form emulsion was judged qualitatively as "good" if the droplets spread in water easily and developed a fine transparent emulsion. It was rated inadequate when there was milky or no emulsion formation, with immediate coalescence of oil droplets. Formulations from F1 to F16 are rapidly forming microemulsions, apparent in appearance and an appropriate SEDDS formulation asset. The grading system identifies it. Results were shown in table 7.

\section{Transmission test}

The clarity of SEDDS was determined through transparency, which was measured in the form of percentage transmittance. It was examined by directly taking the absorbance of the diluted SEDDS. Results present in table 7.

As per table 7, Optimize SMEDSS formulation shows the highest $\%$ transparency, which was $99.8 \%$. Therefore, the value of transmittance is close to $100 \%$. It indicates that each one of the formulations had been transparent. Therefore, the sizes of the globules in all the formulations are withinside the nanometer proportion. In turn, these specify that the drug within the formulations has a large surface area for drug release.

\section{Drug content}

The drug content of all SEDDS formulations was in the proportion of $98.4 \%$ to $99.98 \%$. F5 formulation indicates the highest drug content is $99.98 \%$. Therefore, it indicates good drug distribution in the formulation. Results were shown in table 7.

\section{Determination of self-emulsification time:}

The performance of self-emulsification could be estimated primarily by detecting the rate of emulsification. The SEDDS require dispersing absolutely and rapidly when added to aqueous dilution under slight agitation. The emulsification time of those formulations had been found among the 21 to $30 \mathrm{sec}$. Results had been proven in table 7.

\section{Robustness of dilution}

Robustness to dilution was performed. First, $1 \mathrm{ml}$ of SEDDS was diluted with $1000 \mathrm{ml}$ of water, 0.1N HCL, and acetate buffer pH 4.5. Then, it was stored for 12 hours. All those formulations indicated no precipitation or phase separation after 12 hours. Results were shown in table 8.

\section{Droplet size and polydispersibility index (PDI)}

The droplet size of the microemulsion is a vital parameter in self-emulsification performance. Droplet size needs to be $<100 \mathrm{~nm}$ as it indicates the rate of drug release and absorption. Based on the review, there is no specified limit between SEDDS and SEDDS. The average droplet size found in water varies from 56.45 to 89.58 $\mathrm{nm}$, indicating all the particles were within the nanometer range and homogeneous distribution of size. The tiniest particles were ascertained for formulation F2 $(56.45 \mathrm{~nm})$, and the largest particles were observed for formulations F15. Results were shown in table 7.

\section{Polydispersibility index (PDI)}

Polydispersibility Index indicates the size variety of droplets within the system.

PDI = No. of particles having a size greater than $100 \mathrm{~nm} /$ No. of particles having a size much less than $100 \mathrm{~nm}$. As ideal SEDDS formulation must be widely distributed with particles much less than $100 \mathrm{~nm}$, PDI needs to be less than 0.3. If particles have a size greater than $100 \mathrm{~nm}$, they should be maximum up to $23 \%$. The data (Table 7) shows that most formulations have a PDI less than 0.3 while F3, F4, F9, and F13 have PDI greater than 0.3. The clarity of microemulsion was characterized by transparency, measured in segments of \% transmission. SEDDS forms o/w microemulsion since water is in the external phase. 
Application of Quality by Design Approach to Develop Novel Optimized Self-emulsifying Drug Delivery System of Ezetimibe for Treatment of Poorly Water-soluble Antilipidemic Drug to Enhance its Bioavailability by Using D-optimal Mixture Design

\section{Zeta potential}

Almost all macroscopic materials come in contact with liquid media having an electronic charge on their surfaces. It is a vital indicator of this charge, which may be utilized to predict and control emulsion stability. Due to stable suspension, the charged particles repel every various, conquering the tendency to aggregate. The standard value of Zeta potential is less than $-30 \mathrm{mV}$. It implies that formulations have been stable for a long time. Results are depicted in table 7.

\section{In vitro dissolution study}

In vitro dissolution studies were performed to identify the drug release from the six various formulations (F1-F16) and pure drugs. Dissolution studies were performed for the SEDDS in pH 1.2 buffer solutions. There are no significant differences in the dissolution study of six SEDDS formulations. Around $100 \%$ of the drug is released within 45 to $60 \mathrm{~min}$ in SEDDS, which is greater than the drug release from a pure drug (47.12\%). The dissolution studies were evaluated for $1 \mathrm{hr}$. SEDDS in the spontaneous development of a microemulsion with a small droplet size let in the faster release rate of Ezetimibe in dissolution media. The optimized formulation F2 gives a release in 45 minutes because of the smallest particle size (56.45 nm) and much less PDI value (0.230). The In vitro dissolution research shows that formulations of Ezetimibe with inside SEDDS formulation increase the dissolution properties. In in vitro release study, F2 formulations were optimized, giving $99.98 \%$ release in 45 minutes. Results display in figure 7.

\section{Thermodynamic stability study}

Thermodynamic Stability study determined using temperature and centrifugation. The temperature stability study was carried out by keeping the samples at two different temperatures $\left(2-4^{\circ} \mathrm{C}\right.$ and Room temperature) for 48 hours and performed a visual inspection. Evidence of phase separation, flocculation, and precipitation became determined in the formulation. All formulations are stable at each temperature are shown in Table 8. SEDDS are thermodynamically steady, having a specific centralization of oil, surfactant, and water, with no phase partition, creaming, or breaking. Results display in table 8.

\section{Conclusion}

SEDDS were a promising approach for the formulation of poorly water-soluble drugs. It achieved the predetermined quality char- acteristics of Ezetimibe-SEDDS with the implementation of QbD concepts throughout the development process. We studied the detailed Analysis of the three independent variables called Capmul PG-8, Kolliphor RH 40, Transcutol P and their effects on the quality attributes such as droplet size, emulsification time, \% drug content, and \% drug release with the application of statistical mixture design. This study showed the potential of QbD in SEDDS development. This study successfully developed an optimized Ezetimibeloaded SEDDS formulation using the d-optimal mixture design, a statistical optimization tool based on response surface methodology. The preformulation study of Ezetimibe -loaded SEDDS formulation included Capmul PG-8 (99.73 mg/ml) (oil; X1), Kolliphor RH 40 (57.21 mg/ml) (surfactant; X2), and Transcutol P (135.2 mg/ $\mathrm{ml}$ ) (cosurfactant; X3) and showed excellent in vitro dissolution study. Optimized SEDDS formulation include Capmul PG-8 (18\%,) (oil; X1), Kolliphor RH 40 (40.97\%,) (surfactant; X2), and Transcutol P (40.97\%) (cosurfactant; X3) having particle size (56.45 nm), Polydispersibility index (0.230) zeta potential $(-13.6 \mathrm{mV})$ and the narrow droplet size with uniform distribution. SEDDS formulation showed $99.99 \%$ In vitro release of the drug in 45 minutes compared to the pure drug that demonstrated a $42.12 \%$ drug release like wisely. A good understanding was observed between model prediction and experimental values of percentage drug content (Y1), \% drug release in 20 minutes (Y2), and emulsification time in second (Y3), and droplet size in nm (Y4). Thus, the finding shows that optimizing Ezetimibe-loaded SEDDS formulation is potentially used to improve the oral absorption of poorly water-soluble drugs.

\section{Conflict of Interest}

The authors declare that they have no known competing personal and no financial interests that could have appeared to influence the work reported in this paper. The authors alone are responsible for the content and writing of this article.

\section{Bibliography}

1. Gahlawat V., et al. "Application of D-optimal Mixture Design for Development and Optimization of Olmesartan Medoxomil Loaded SEDDS". Current Drug Therapy 15 (2020): 548-560.

2. Carlos T., et al. "Improvement in the oral bioavailability and efficacy of new ezetimibe formulations- Comparative study of solid dispersion and different micellar systems". Pharmaceutics 12 (2020): 617. 
3. Yeom DW., et al. "Development and optimization of a selfmicro emulsifying drug delivery system for atorvastatin calcium using d-optimal mixture design". International Journal of Nanomedicine 10 (2015): 3865-3878.

4. Karavasili C., et al. "Self-Nanoemulsifying Drug Delivery Systems (SNEDDS) Containing Rice Bran Oil for Enhanced Fenofibrate Oral Delivery: In-Vitro Digestion, Ex Vivo Permeability, and In-Vivo Bioavailability Studies". AAPS Pharmaceutical Science and Technology 21 (2020): 1765-1972.

5. Dash R., et al. "Design, optimizes and evaluates glipizide solid self-nano emulsifying drug delivery for enhanced solubility and dissolution". Saudi Pharmaceutical Journal 23.5 (2015): 528-540.

6. Patel M., et al. "Self-micro emulsifying drug delivery system of lurasidone hydrochloride for enhanced oral bioavailability by lymphatic targeting: In-Vitro, Caco-2 cell line In-Vivo evaluation". International journal of Pharmaceutical Science 138 (2019): 105027.

7. Patel MS., et al. "Microemulsion Based Gel: A Review". International Journal of Universal Pharmacy and Biomedical Sciences 3 (2014): 63-78.

8. Kavitha AN., et al. "A Structural Framework for Developing SEDDS through Quality by Design Approach". Asian Journal of Pharmaceutics 14 (2020): 308-318.

9. Kavitha AN., et al. "Quality by design-based development of etravirine self-micro emulsifying drug delivery system". International Journal of Applied Pharm 13 (2021): 103-111.

10. Holm R., et al. "Optimization and self-micro emulsifying drug delivery system (SEDDS) using a D-optimal design and the desirability function". Drug Development and Industrial Pharmacy 32 (2006): 1025-1032.

11. Son HY., et al. "Optimization of self-micro emulsifying drug delivery system for phospholipid complex of Telmisartan using D optimal mixture design". PLoS ONE 13 (2018): e0208339.

12. Kavitha AN., et al. "Design and Development of Darunavir loaded SEMDDS using Extreme Vertices Mixture Design in a Quality by Design Framework". Industrial Journal of Pharmaceutical Education and Research 54 (2020): 337-3348.

13. Gowthami K., et al "Quality by Design based Development of Self Nano Emulsifying Drug Delivery System of Ritonavir". Journal Young Pharmacist 12 (2020): 215-220.
14. Sarwar., et al. "QbD-based systematic development of novel optimized solid self-nano emulsifying drug delivery systems (SNEDDS) of lovastatin with enhanced biopharmaceutical performance". Drug Delivery 22 (2014): 765-784.

15. Gardouh AR., et al. "Design and evaluate combined Atorvastatin and Ezetimibe optimized self-nano emulsifying drug delivery system". Journal of Drug Delivery Science and Technology 60 (2020): 102093.

16. Huo T., et al. "Preparation and comparison of tacrolimus-loaded solid dispersion and Self-Microemulsifying Drug Delivery System by In-Vitro/In-Vivo Evaluation". European Journal of Pharmaceutical Science 114 (2017): 74-83.

17. Huang W., et al. "Enhanced anticancer effect of Brucea javanica oil by solidified self-micro emulsifying drug delivery system". Journal of Drug Delivery Science and Technology 48 (2018): 266-273.

18. Kantaporn K., et al. "Development and characterization of clove oil nanoemulsions and self- micro emulsifying drug delivery systems". Journal of Drug Delivery Science and Technology 46 (2018): 330-338.

19. Kamboj S., et al. "Quality-by-design based development of a self-micro emulsifying drug delivery system to reduce food effect of Nelfinavir mesylate". International Journal Pharmaceutical Science 30 (2016): 311-325.

20. Akiladevi D., et al. "Preparation and In Vitro Evaluation of SelfEmulsifying Drug Delivery System for Oral Delivery of Glibenclamide". International Journal of Pharmacy and Pharmacology 1 (2017): 105-112.

21. Kazi M., et al. "Enhancing Oral Bioavailability of Apigenin Using a Bioactive Self-Nano Emulsifying Drug Delivery System (Bio-SNEDDS): In-Vitro, In-Vivo, and Stability Evaluations". Pharmaceutics 12 (2020): 749.

22. Li S. "Effect of ionization of drug-on-drug solubilization in SEDDS prepared using Capmul MCM and caprylic acid". Asian Journal of Pharmaceutical Science 2 (2017): 73-82.

23. Liu H., et al. "Improving the Oral Absorption of Nintedanib By A Self-Microemulsion Drug Delivery System: Preparation and In-Vitro/In-Vivo Evaluation". International Journal of Nanotechnology 14 (2019): 8739-8751. 
24. Mundada VP., et al. "Enhanced Oral Bioavailability and Anticoagulant Activity of Dabigatran Etexilate by Self Microemulsifying Drug Delivery System: Systematic Development, In-vitro, Ex vivo, and In-vivo Evaluation". Journal of Nanomedicine and Nanotechnology 9 (2018): 480.

25. Nan., et al. "Formulation and evaluation of luteolin supersaturate self nano emulsifying drug delivery system (S-SNEDDS) for enhanced oral bioavailability". Journal of Drug Delivery Science and Technology 58 (2020): 101783.

26. Pramanik S., et al. "Development of Solid Self-Microemulsifying System of Tizanidine Hydrochloride for Oral Bioavailability Enhancement: In-Vitro and In-Vivo Evaluation". AAPS Pharmaceutical Science and Technology 21 (2020): 182.

27. Reddy MS., et al. "Formulation and in-vitro characterization of self-micro emulsifying drug delivery systems of rivaroxaban". International Journal Pharmaceutical Science and Research 8 (2017): 3436-3445.

28. Ratnaparkhi MP., et al. "Design, Development, and Characterization of Self Micro Emulsifying Drug Delivery System for Aceclofenac". International Journal of Pharmaceutical Science and Research 9 (2018): 3815-3826.

29. Sahoo S., et al "Design and Development of Self Microemulsifying Drug Delivery Systems (SEDDS) of Telmisartan for Enhancement of In-Vitro Dissolution and Oral Bioavailability in Rabbit". International Journal of Applied Pharma 10 (2018): 117-126.

30. Wagh MP., et al. "Solid self-emulsifying drug delivery system: preparation technique and dosage forms". International Journal of Biopharmaceutics 5 (2014): 101-108.

31. Wang CY., et al. "Self-Nano emulsifying Drug Delivery Systems for Enhancing Solubility, Permeability, and Bioavailability of Sesamin". Molecules 25 (2020): 3119.

\section{Volume 5 Issue 8 August 2021}

\section{(C) All rights are reserved by Mukesh S Patel., et al.}

